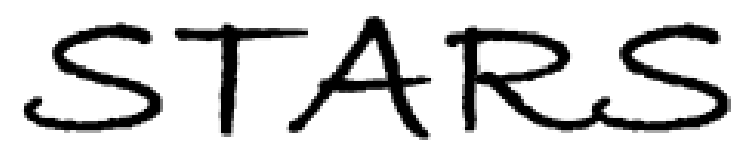

University of Central Florida

STARS

$1-1-2005$

\title{
Oceanic rain rate estimates from the QuikSCAT Radiometer: A Global Precipitation Mission pathfinder
}

\author{
Khalil A. Ahmad \\ University of Central Florida \\ W. Linwood Jones \\ University of Central Florida \\ Takis Kasparis \\ University of Central Florida \\ Stephen Wiechecki Vergara \\ University of Central Florida \\ Ian S. Adams \\ University of Central Florida \\ Find similar works at: https://stars.library.ucf.edu/facultybib2000 \\ University of Central Florida Libraries http://library.ucf.edu \\ See next page for additional authors
}

This Article is brought to you for free and open access by the Faculty Bibliography at STARS. It has been accepted for inclusion in Faculty Bibliography 2000s by an authorized administrator of STARS. For more information, please contactSTARS@ucf.edu.

\section{Recommended Citation}

Ahmad, Khalil A.; Jones, W. Linwood; Kasparis, Takis; Vergara, Stephen Wiechecki; Adams, Ian S.; and Park, Jun D., "Oceanic rain rate estimates from the QuikSCAT Radiometer: A Global Precipitation Mission pathfinder" (2005). Faculty Bibliography 2000s. 4948.

https://stars.library.ucf.edu/facultybib2000/4948

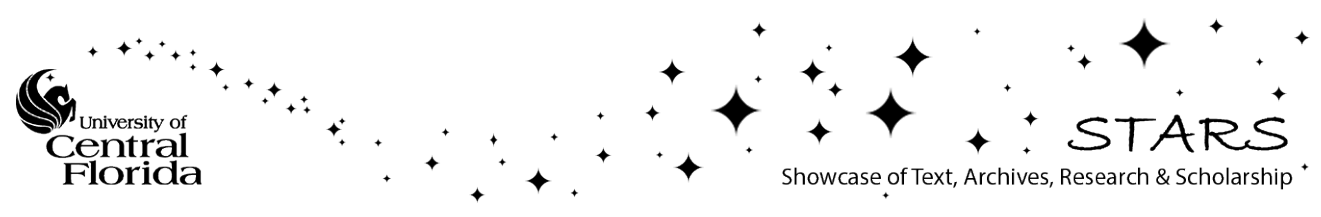




\section{Authors}

Khalil A. Ahmad, W. Linwood Jones, Takis Kasparis, Stephen Wiechecki Vergara, Ian S. Adams, and Jun D. Park 


\title{
Oceanic rain rate estimates from the QuikSCAT Radiometer: A Global Precipitation Mission pathfinder
}

\author{
Khalil A. Ahmad, W. Linwood Jones, Takis Kasparis, Stephen Wiechecki Vergara, \\ Ian S. Adams, and Jun D. Park \\ Central Florida Remote Sensing Laboratory, Department of Electrical and Computer Engineering, University of Central
} Florida, Orlando, Florida, USA

Received 29 October 2004; revised 13 February 2005; accepted 24 February 2005; published 1 June 2005.

[1] The SeaWinds scatterometer, launched onboard the QuikSCAT satellite in 1999, measures global ocean vector winds. In addition to measuring radar backscatter, SeaWinds simultaneously measures the microwave brightness temperature of the atmosphere/surface, and this passive microwave measurement capability is known as the QuikSCAT Radiometer (QRad). This paper presents a QRad retrieval algorithm used to infer instantaneous oceanic rain rates. This statistical algorithm is trained using near-simultaneous observations of major rain events by QRad and the Tropical Rainfall Measuring Mission (TRMM) Microwave Imager (TMI). Rain rate retrieval algorithm validation is presented through comparisons with independent rain measurements from the TMI 2A12 surface rain rates and the TRMM 3B42RT composite microwave and visible and infrared near-real time data product. Results demonstrate that QRad rain rate measurements are in good agreement with these independent microwave rain observations and superior to the visible/infrared rain estimates. Thus the QRad rain measurement time series is a valuable addition to the oceanic precipitation climatology that can be used to improve the diurnal estimation of the global rainfall, which is a goal for the future Global Precipitation Mission program. Moreover, the availability of QRad data will provide GPM users early access to learn to use less-precise rain measurements that will occur in the GPM era with the use of less-capable constellation satellites. Finally, these QRad rain estimates will be available in the planned data reprocessing (FY 2006) of QuikSCAT winds to improve the rain flagging of rain-contaminated oceanic wind vector retrievals.

Citation: Ahmad, K. A., W. L. Jones, T. Kasparis, S. W. Vergara, I. S. Adams, and J. D. Park (2005), Oceanic rain rate estimates from the QuikSCAT Radiometer: A Global Precipitation Mission pathfinder, J. Geophys. Res., 110, D11101, doi:10.1029/2004JD005560.

\section{Introduction}

[2] For more than one decade, multi-frequency microwave radiometer imagers flying on low earth satellites have provided valuable day/night remote sensing of oceanic and atmospheric variables; but the emphasis on oceanic precipitation measurements achieved a significant advance with the launch of the Tropical Rainfall Measuring Mission (TRMM) observatory in late 1997. Because of TRMM's non-sun synchronous orbit, for the first time, precipitation measurements were available from a satellite over all local times so that the diurnal cycle of oceanic precipitation could be studied. However, from 1998 through late 2002, the ocean sampling was very sparse with only four such satellite instruments operating on-orbit; three Defense Meteorological Support Program (DMSP) satellites carrying the Special

Copyright 2005 by the American Geophysical Union. 0148-0227/05/2004JD005560
Sensor Microwave Imager (SSM/I), and the Tropical Rainfall Measuring Mission's (TRMM) Microwave Imager (TMI). The SSM/I's fly on near-polar sun synchronous satellites that provide greater than $90 \%$ earth coverage daily; however, since they fly in a day/night terminator orbit, they provide only morning and evening sampling times. On the other hand, the TMI flies in a low inclination $\left(38^{\circ}\right)$ non-sun synchronous orbit that has been optimized to measure tropical rainfall. TMI provides full diurnal sampling over the period of slightly greater than one month. However, even with the four passive microwave sensors, the statistics of oceanic rainfall were badly under-sampled. Since the fall of 2002, a fifth microwave imager, the Advanced Microwave Scanning Radiometer (AMSR-E) on NASA's Aqua earth observing system satellite began its ocean precipitation measurements; but even with this additional radiometer, the diurnal sampling is still less than desired.

[3] Many researchers [e.g., Wilheit et al., 1991; Petty and Katsaros, 1992; Bell and Reid, 1993; Chang et al., 1995; Imaoka and Spencer, 2000] have studied diurnal sampling 


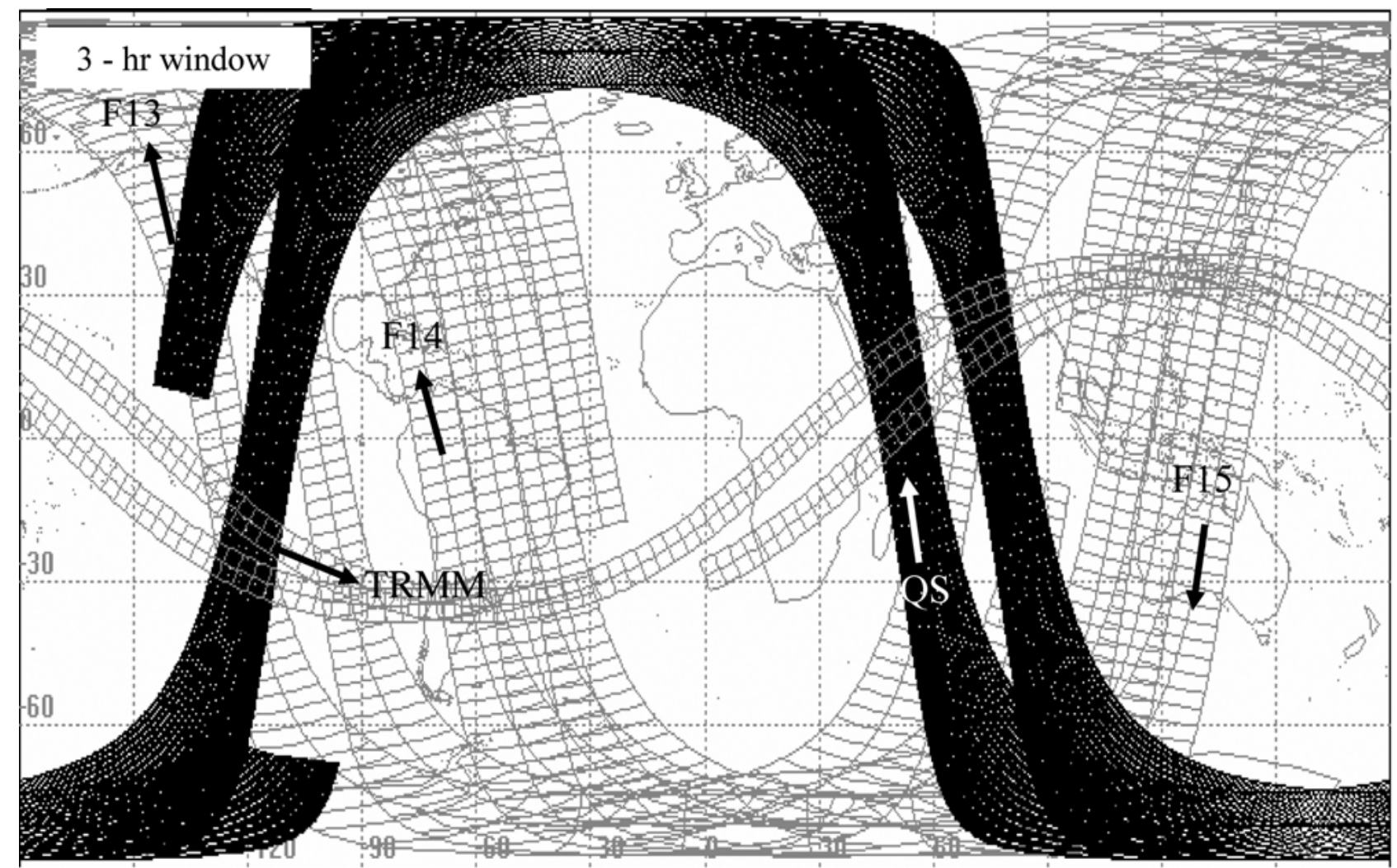

Figure 1. Three hour sampling provided by 3-SSMI's (F-13, F-14 and F-15), TRMM Microwave Imager and QuikSCAT Radiometer swaths for time window, 0-3 hours Zulu, on March 1, 2000.

of oceanic precipitation using satellite microwave radiometers. Because of the sparse sampling, diurnal cycles must be estimated using large space-time averages, and likewise, it is difficult to determine the rainfall statistics for regional oceanic precipitation. In the future, a constellation of satellites, known as the Global Precipitation Mission (GPM) [Smith, 2001; Smith et al., 2001], will solve this observational shortage. This proposed constellation, comprised of satellites in low inclination and polar low-earth orbits (non-sun synchronous and sun synchronous) will provide near-global coverage with a worst case revisit time of three hours at the equator. An important aspect of GPM is the use of a highly capable "core observatory" (similar to TRMM) to provide rainfall classification and rain rate retrievals. This will be augmented by six or more less-capable "constellation" satellites carrying microwave radiometers, which are cross-calibrated to the core observatory, and provide the rapid temporal sampling of rainfall. Thus, in the future, scientists and operational users will have to learn to accommodate rain retrievals of varying quality in their research and applications.

[4] In September 1999, the QuikSCAT Radiometer (QRad) began ocean precipitation measurements, which provides additional independent samples over SSM/I and TMI. A typical example of the QRad sampling is shown in Figure 1 for a three-hour window (universal time: 00:0003:00). Also shown are the corresponding sampling coverage for TMI and three SSM/I's. It is observed that QRad increases the coverage area by about $10 \%$; but even with five microwave imagers, the ocean sampling is still only approximately $60 \%$ in a typical 3-hour window. Never the less, the QRad's sampling contribution is significant in that the daily average revisit time is reduced as shown in Figure 2. For clarity of presentation, sampling improve-

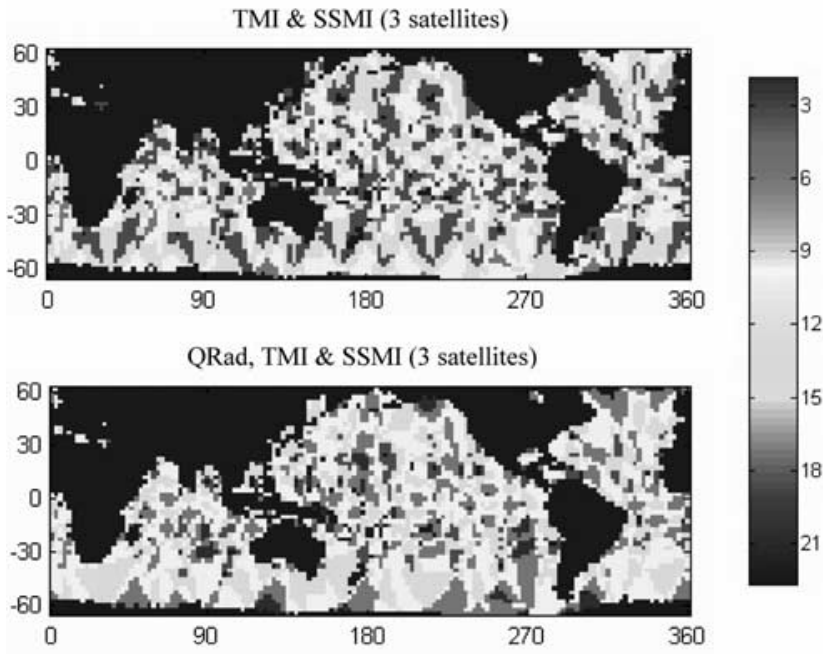

Figure 2. Ocean sampling, daily average revisit time. (top) TMI and 3-SSMI's and (bottom) sampling with QRad added. See color version of this figure at back of this issue. 


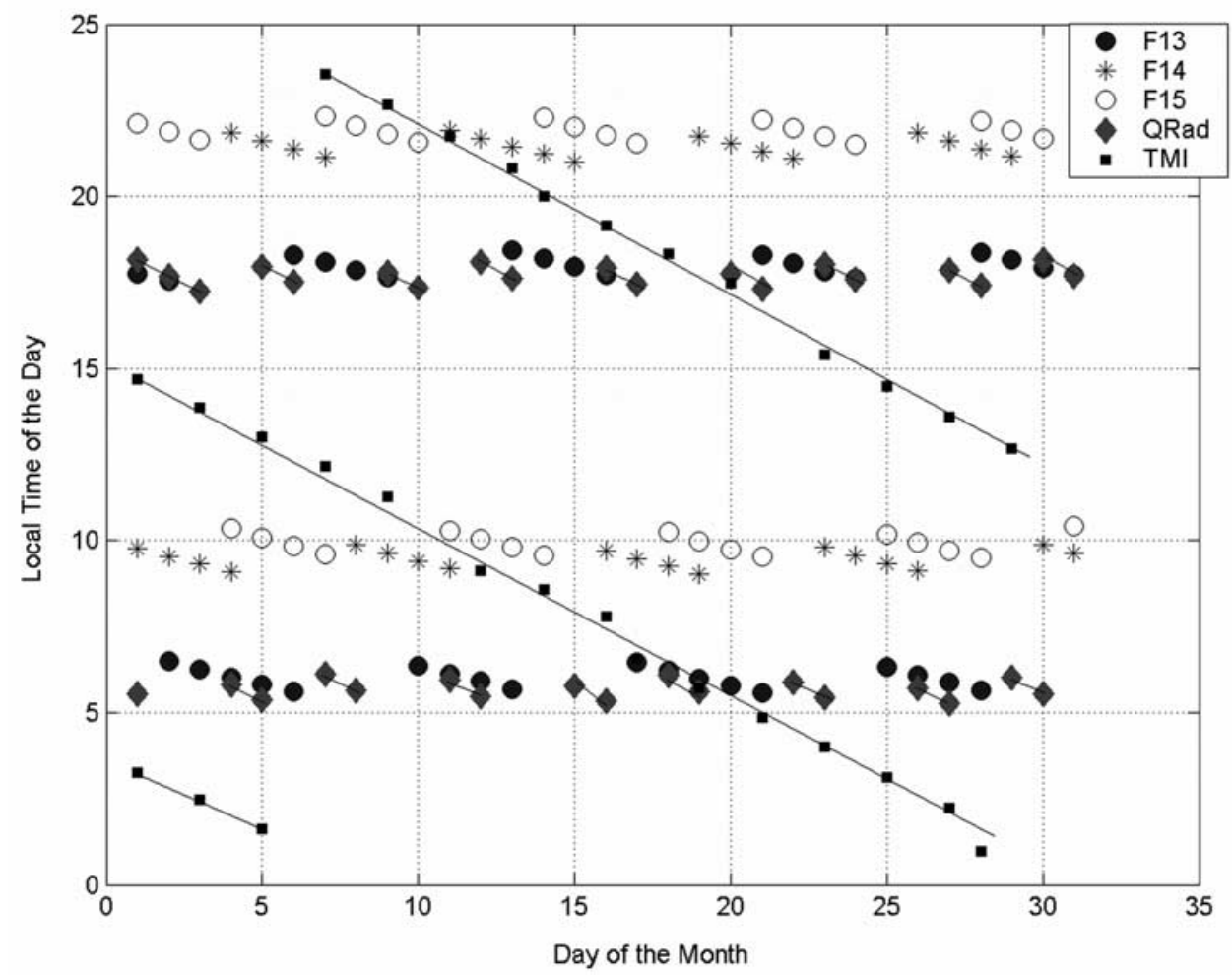

Figure 3. Typical time of day sampling for SSMI (F-13, F-14 and F-15), TMI and QuikSCAT Radiometer. Sample location is $1^{\circ} \times 1^{\circ}$ latitude/longitude box located at equator and prime meridian.

ments, due to the QRad and averaged over $20^{\circ}$ latitudinal zones, are quantitatively summarized in Table 1. Further, an additional illustration of QRad oceanic sampling contribution is shown in Figure 3 which presents a typical scenario of "local time of day" QRad sampling over a $1^{\circ} \times 1^{\circ}$ box located at equator and prime meridian for a period of one month. Also shown are the local time samplings for TMI and three SSM/I instruments. It is clear that $\mathrm{QRad}$ is providing independent sampling which complements and fills in the gaps between the sampling times of the other satellites.

[5] Thus the QRad time series (from September 1999 to present) is a valuable addition to the ocean precipitation

Table 1. Average Oceanic Coverage in a Typical 3-Hour Window $^{\text {a }}$

\begin{tabular}{cccc}
\hline & $\begin{array}{c}\text { Ocean } \\
\text { Coverage } \\
\text { Without } \\
\text { QRad }\end{array}$ & $\begin{array}{c}\text { Ocean } \\
\text { Coverage } \\
\text { With } \\
\text { Qrad }\end{array}$ & $\begin{array}{c}\text { QRad } \\
\text { Contribution }\end{array}$ \\
\hline $40^{\circ} \mathrm{N}-60^{\circ} \mathrm{N}$ & $57.94 \%$ & $68.55 \%$ & $10.61 \%$ \\
$20^{\circ} \mathrm{N}-40^{\circ} \mathrm{N}$ & $58.23 \%$ & $64.69 \%$ & $6.46 \%$ \\
$0^{\circ}-20^{\circ} \mathrm{N}$ & $63.70 \%$ & $71.34 \%$ & $7.64 \%$ \\
$20^{\circ} \mathrm{S}-0^{\circ}$ & $63.13 \%$ & $70.17 \%$ & $7.04 \%$ \\
$40^{\circ} \mathrm{S}-20^{\circ} \mathrm{S}$ & $57.69 \%$ & $63.87 \%$ & $6.18 \%$ \\
$60^{\circ} \mathrm{S}-40^{\circ} \mathrm{S}$ & $58.34 \%$ & $68.26 \%$ & $9.93 \%$ \\
\hline
\end{tabular}

${ }^{\mathrm{a}}$ Improvements due to QRad contribution are calculated for regions of $20^{\circ}$ latitudinal zones. climate data set. Further, the early availability of QRad rain measurements provides an excellent opportunity for learning how to utilize future GPM data sets. As will be described, even though the quality of the QRad rain retrievals are somewhat limited compared to TMI and $\mathrm{SSM} / \mathrm{I}$, they certainly are useful in that they provide additional temporal/spatial sampling. Moreover, they provide simultaneous, collocated precipitation measurements with QuikSCAT ocean surface wind vectors for rainflagging contaminated wind vector retrievals.

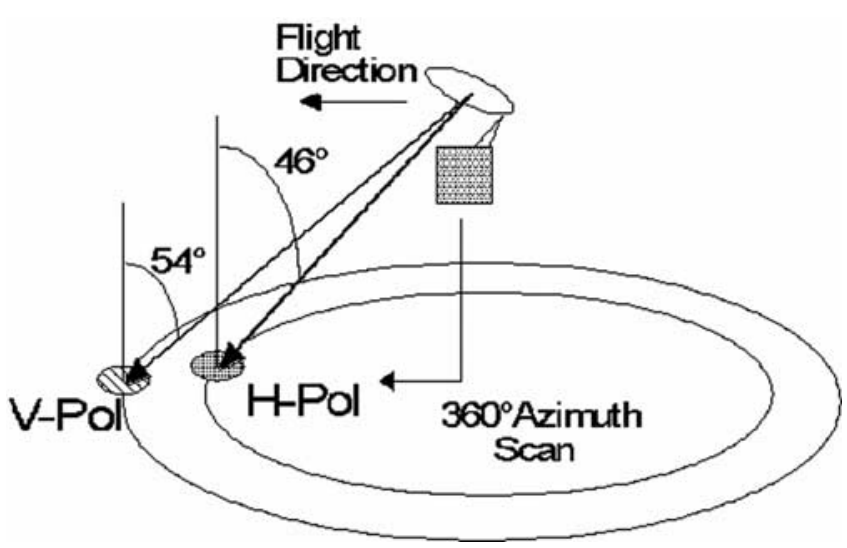

Figure 4. Measurement geometry of SeaWinds instrument onboard QuikSCAT satellite. 

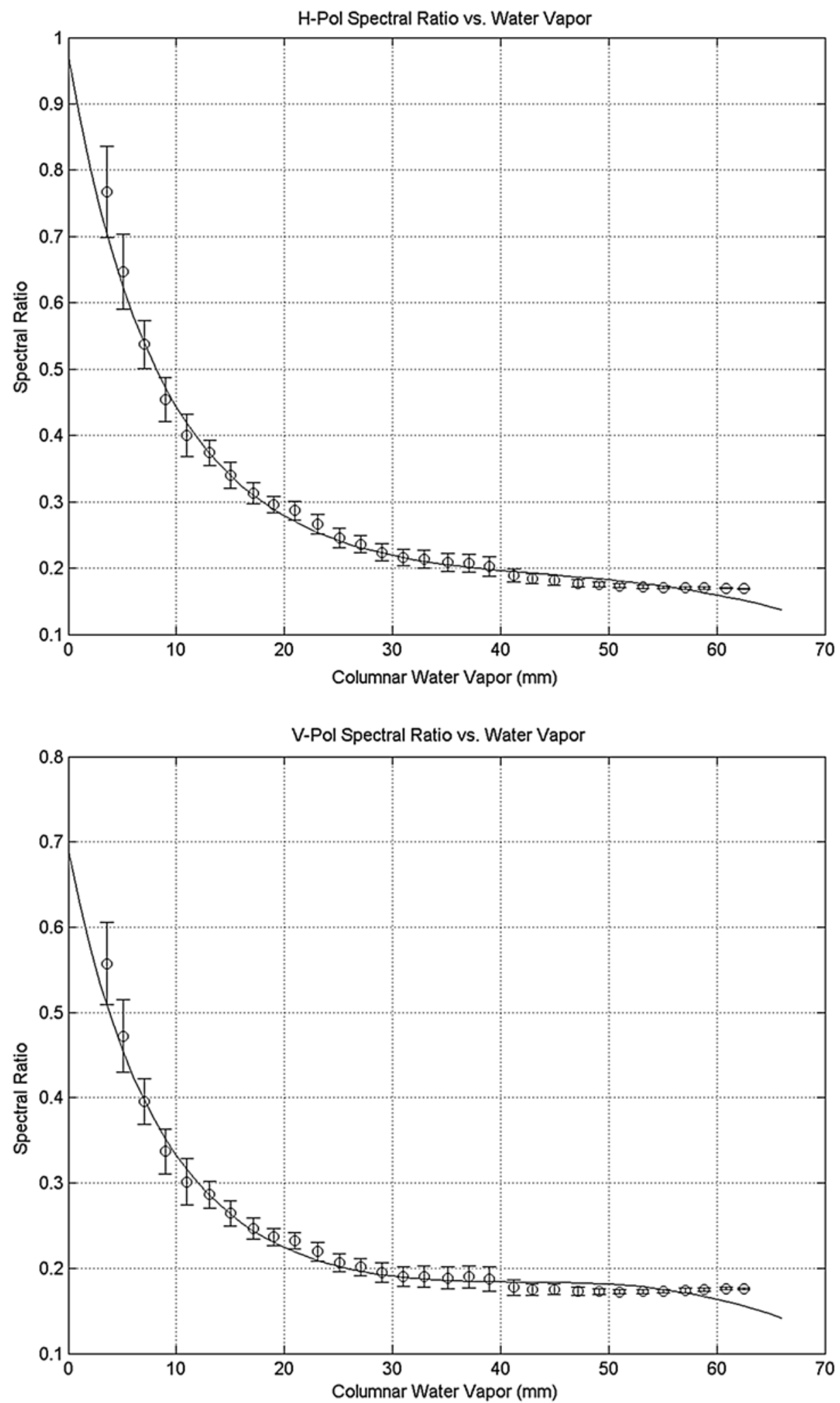

Figure 5. Brightness temperature spectral ratio as a function of columnar water vapor. (top) A plot of horizontal polarization and (bottom) the vertical polarization. Circles denote binned/averaged data and the error bars show \pm one standard deviation. The solid line shows the third order polynomial fit. 


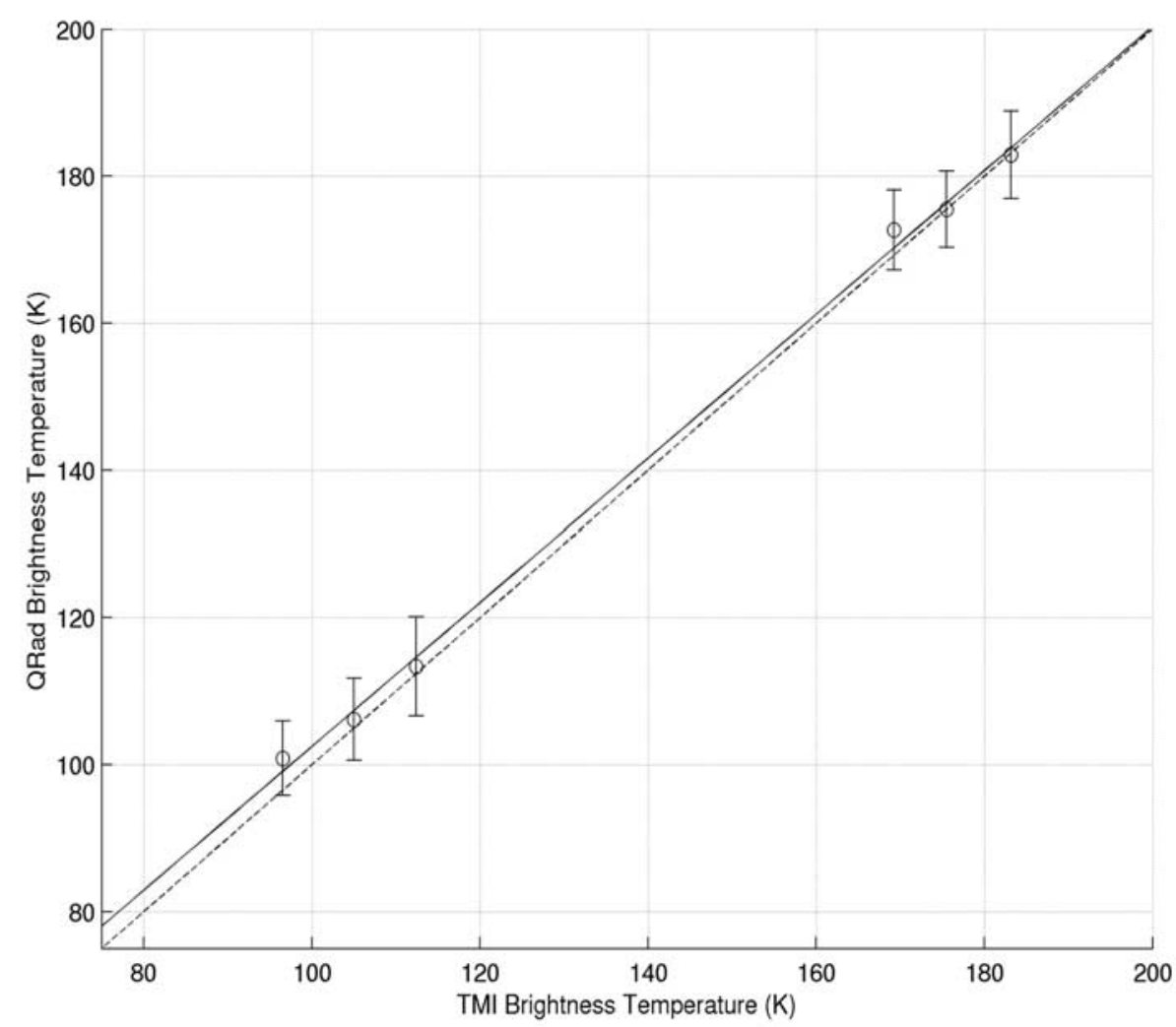

Figure 6. Comparison of QRad and TMI ocean brightness temperatures for rain-free five day averages. Circles are binned/averaged data, and error bars represent \pm one standard deviation. Dashed line is perfect agreement and solid line shows least squares regression.

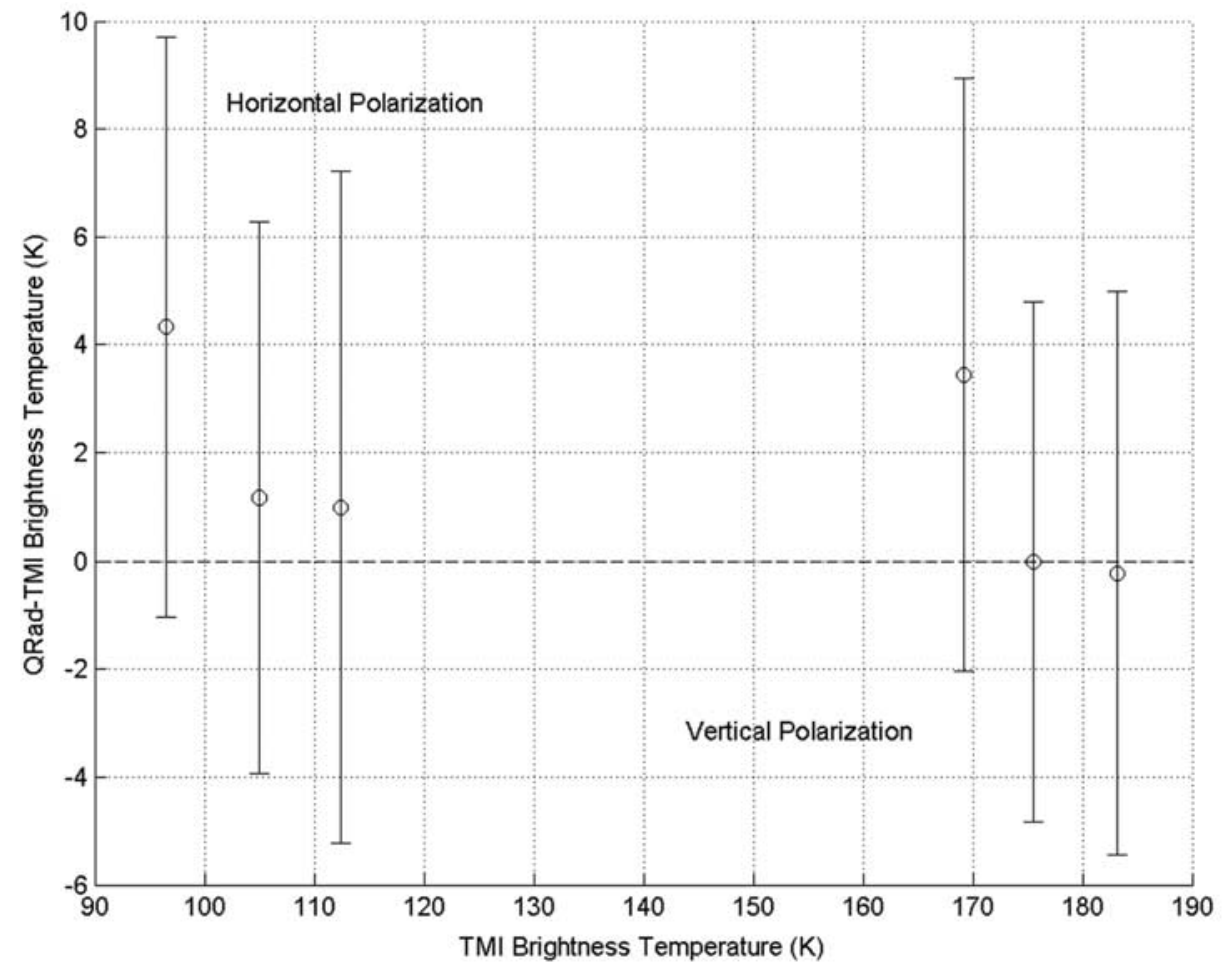

Figure 7. Five-day average oceanic brightness temperature differences (QRad - TMI) for rain-free ocean, April 2003. Circles are binned/averaged in $5 \mathrm{~K}$ bins by TMI, and error bars denote \pm one standard deviation. 
Table 2. Linear Fit of QRad to TMI Ocean Brightness Temperatures $^{\mathrm{a}}$

\begin{tabular}{lcc}
\hline \multicolumn{1}{c}{ Date } & Offset & Slope \\
\hline Sept. 1999 & $6.55 \mathrm{~K}$ & 0.977 \\
June 2000 & $6.32 \mathrm{~K}$ & 0.955 \\
Jan. 2001 & $9.07 \mathrm{~K}$ & 0.958 \\
April 2003 & $4.67 \mathrm{~K}$ & 0.978 \\
\hline
\end{tabular}

${ }^{\mathrm{a}}$ Data are rain-free combined horizontal and vertical polarization threeday averaged ocean brightness temperatures. TMI brightness temperatures are interpolated to QRad frequency and extrapolated to QRad incidence angle.

[6] In this paper, the oceanic rain measurements made with QRad are described. Section 2 describes the QuikSCAT instrument and the external radiometric calibration procedure. The QRad rain rate algorithm is discussed in detail in section 3; and validation of QRad rain measurements through comparisons with other independent rain measuring instruments are presented in section 4. Results demonstrate that the QRad oceanic rain estimates are in good agreement with TMI and SSMI independent rain measurements.

\section{QuikSCAT Radiometer}

\subsection{Instrument Description}

[7] The SeaWinds scatterometer on the QuikSCAT satellite is a conical scanning long-pulse radar system used to measure the backscatter from the ocean surface to infer surface wind speed and direction [Spencer et al., 1997]. This scatterometer has two receiver channels, which allow the received backscatter signal (echo) and the black-body microwave emission (noise) from the ocean surface and interviewing atmosphere to be separated. Although, quanti- tative microwave brightness temperature measurements were not originally envisioned; never the less, the QuikSCAT radiometric function has been implemented post-launch through ground signal processing. Thus QRad measures the linearly polarized microwave brightness temperature $T_{b}$, at $13.4 \mathrm{GHz}$ using a mechanical spinning reflector antenna as shown in Figure 4. Microwave emissions are collected over the entire conical scan (forward and aft looking) with separate offset "pencil beams" at $46^{\circ}$ incidence (horizontal polarization, H-pol) and $54^{\circ}$ incidence (vertical polarization, V-pol). Individual Tb's are averaged on a spacecraft measurement grid of wind vector cells at $25 \mathrm{~km}$ resolution that results in mean horizontal and vertical $\mathrm{T}_{\mathrm{b}}$ 's collocated with the normalized backscatter measurements. The pulse repetition frequency and antenna scan rate have been designed to provide approximately $50 \%$ overlap of the instantaneous field of view (IFOV) in both the along track and cross track directions. Thus with the two pencil beams, it is possible to isolate the microwave emissions from the earth into elliptical footprints defined by the oneway antenna pattern half-power contours (approximately $35 \mathrm{~km} \times 50 \mathrm{~km}$ ). Details of the QRad instrument and its radiometric calibration are provided by Jones et al. [2000] and Mehershahi [2000].

\subsection{Radiometric Calibration}

[8] Designed as a radar, SeaWinds is not an optimum radiometer. Brightness temperatures $\left(\mathrm{T}_{\mathrm{b}}\right.$ 's) are calculated for each received pulse with an equivalent integration time of $1.5 \mathrm{~ms}$ and a noise bandwidth of only $750 \mathrm{KHz}$. Because of the limited time-bandwidth product, the radiometric precision is much lower than desired $(\Delta \mathrm{T}=27 \mathrm{Kelvin} / \mathrm{pulse})$. For QRad rain measurements, this can be partially ameliorated by using spatial and temporal averaging where both for-

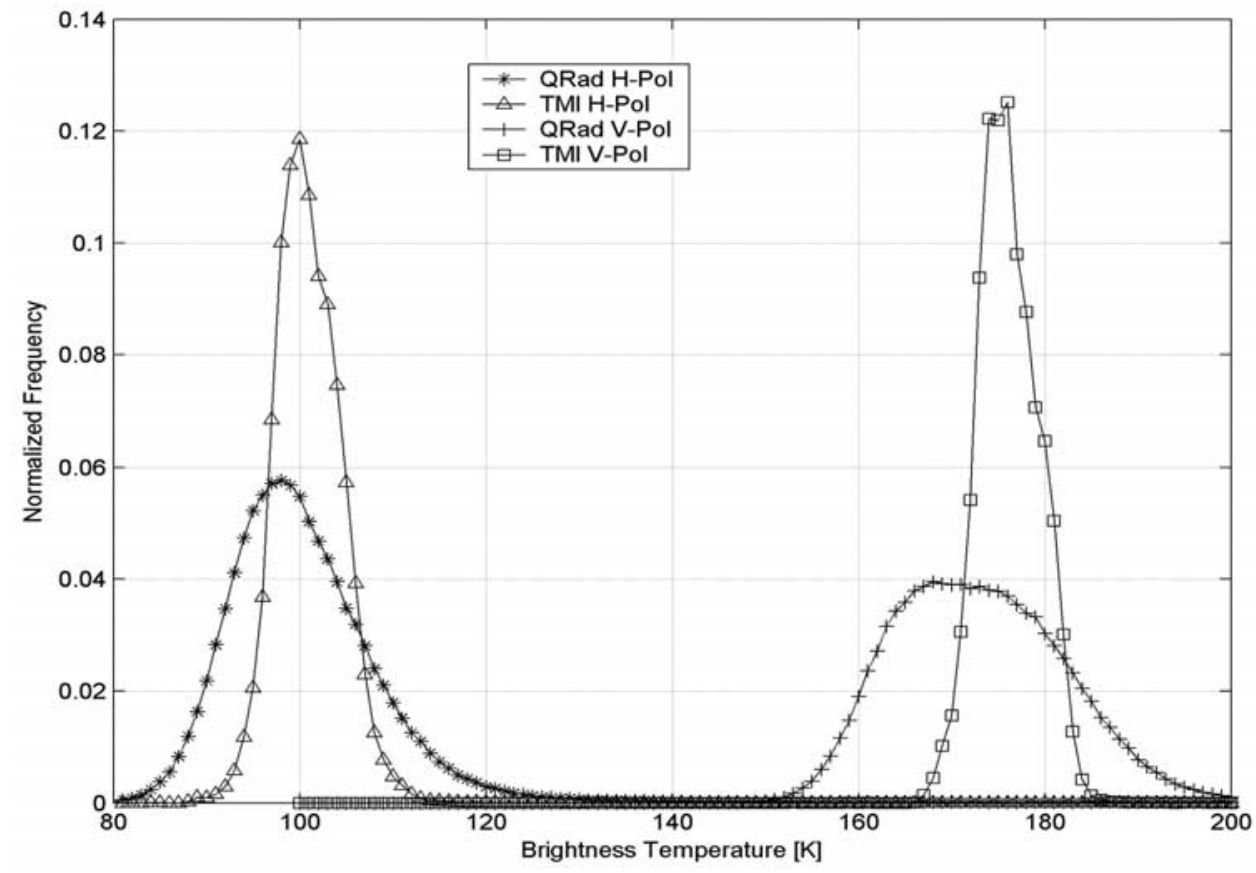

Figure 8. Three-day average, rain-free, ocean brightness temperature probability density function, January $15-17,2000$. 
Table 3. Median Seasonal Ocean Brightness Temperatures for Year $2000^{\mathrm{a}}$

\begin{tabular}{lcccc}
\hline \multicolumn{1}{c}{ Date } & Qrad (H-pol) & TMI (H-pol) & Qrad (V-pol) & TMI (V-pol) \\
\hline January & 99.4 & 100.7 & 172.6 & 175.9 \\
March & 101.1 & 101.5 & 173.1 & 176.4 \\
April & 100.0 & 101.2 & 172.7 & 176.5 \\
July & 101.2 & 101.1 & 173.7 & 175.7 \\
September & 100.3 & 100.4 & 173.7 & 175.6 \\
October & 100.3 & 100.4 & 173.7 & 175.6 \\
\hline
\end{tabular}

${ }^{\mathrm{a}}$ Brightness temperatures are rain-free three-day average. TMI brightness temperatures are interpolated to QRad frequency and extrapolated to QRad incidence angle.

ward-looking and aft-looking azimuth directions are collocated onto a $0.5^{\circ} \times 0.5^{\circ}$ earth-located grid that is approximately equivalent to the QRad antenna surface resolution $(50-\mathrm{km})$. Each polarized $\mathrm{T}_{\mathrm{b}}$ observation is the average of about 24 pulses that results in a $\Delta \mathrm{T}=5 \mathrm{~K}$.

[9] Unfortunately for QRad there are no provisions for the usual two-point, hot and cold, absolute brightness temperature calibration. However, the QRad radiometric gain calibration is accomplished once per antenna scan using an internal ambient temperature (warm) load in the receiver; and the $\mathrm{T}_{\mathrm{b}}$ offset is established one time, in an onorbit calibration in 2000, using external comparisons with a well-known natural black-body sources (the Amazon rain forest) and with selected rain-free ocean $\mathrm{T}_{\mathrm{b}}$ measurement comparisons with TMI.

[10] For the ocean calibration, rain-free QRad polarized $\mathrm{T}_{\mathrm{b}}$ 's are averaged for 3-days and are spatially collocated with TMI brightness measurements (over $\pm 40^{\circ}$ latitude on a $0.25^{\circ}$ latitude $\times 0.25^{\circ}$ longitude grid). Because the polarized ocean $T_{b}$ 's change with frequency and because TMI does not have a $13.4 \mathrm{GHz}$ channel, a translation of TMI brightness temperatures must be performed before direct comparisons are possible with QRad. For TMI, the two lowest frequency channels (10.7 and $19.4 \mathrm{GHz})$ bracket the QRad frequency at $13.4 \mathrm{GHz}$; however, the incidence angles do not match. The TMI incidence angle is $52.8^{\circ}$ for all channels; whereas, for QRad, the inner (H-pol) beam is $46^{\circ}$ and the outer ( $\mathrm{V}$-pol) beam is $54^{\circ}$. Thus, as described below, TMI $\mathrm{T}_{\mathrm{b}}$ 's are interpolated over frequency and extrapolated over incidence angle to create $\mathrm{QRad}$ equivalent $\mathrm{T}_{\mathrm{b}}$ 's, which are used to establish the QRad absolute radiometric offset.

[11] Over oceans, a microwave radiative transfer model developed by Wisler and Hollinger [1977] is used to calculate the theoretical $\mathrm{T}_{\mathrm{b}}$ 's for both QRad and TMI channels. At vertical polarization, QRad and TMI measurements are at similar incidence angles; thus TMI measurements are mostly frequency interpolated with a small incidence angle correction. For horizontal, the $7^{\circ}$ difference in incidence angle requires a significant incidence angle adjustment as well as frequency interpolation. Using the radiative transfer model, theoretical $T_{b}$ values are used to determine a non-linear interpolation, thus producing the equivalent QRad Tb's from TMI observations at 10.7 and $19 \mathrm{GHz}$.

$$
T b_{13.4}=T b_{10.7}+s r\left(T b_{19.4}-T b_{10.7}\right)
$$

where $s r$ is a "spectral ratio," defined as:

$$
s r=\frac{T b_{13.4}-T b_{10.7}}{T b_{19.4}-T b_{10.7}}
$$

[12] Analysis has shown that this spectral ratio yields equivalent QRad $\mathrm{T}_{\mathrm{b}}$ 's accurate to within a few Kelvin [Mehershahi, 2000; Jones et al., 2000]. However, for the given frequencies, this spectral ratio exhibits a nearly exponential dependence on atmospheric columnar water

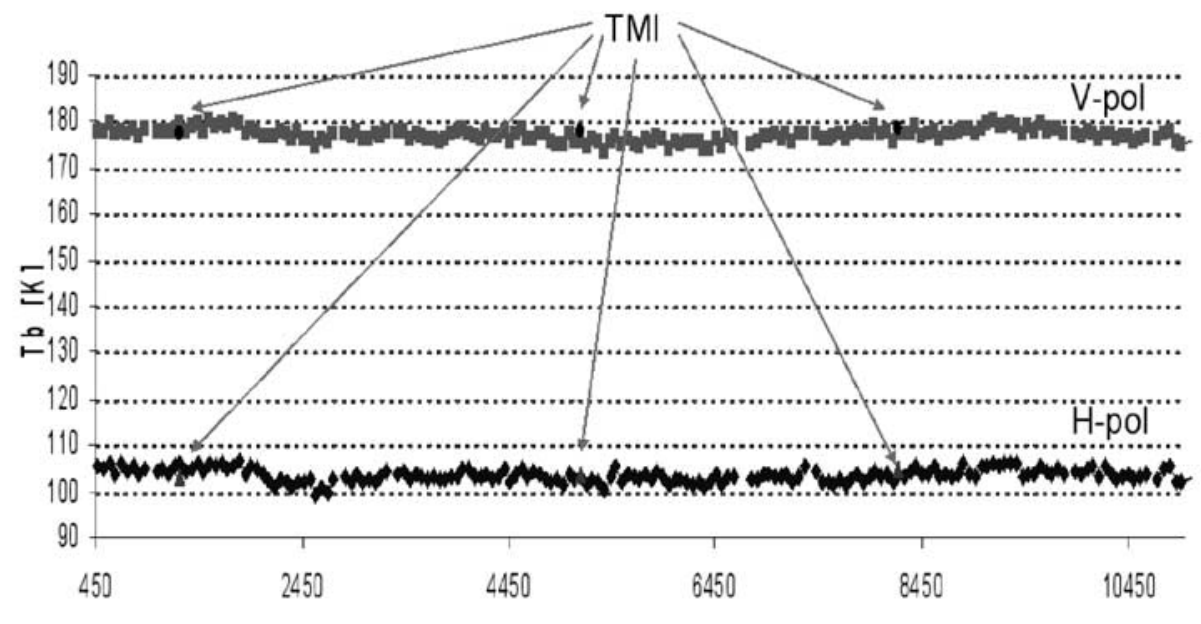

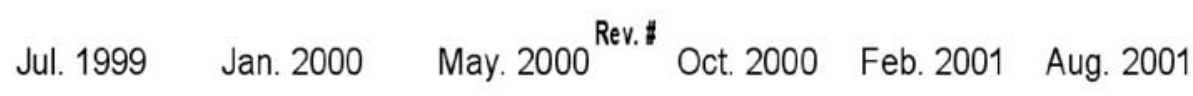

Figure 9. Pacific Ocean brightness temperature time series from QRad for repeating ground swath at approximately four-day sampling. 


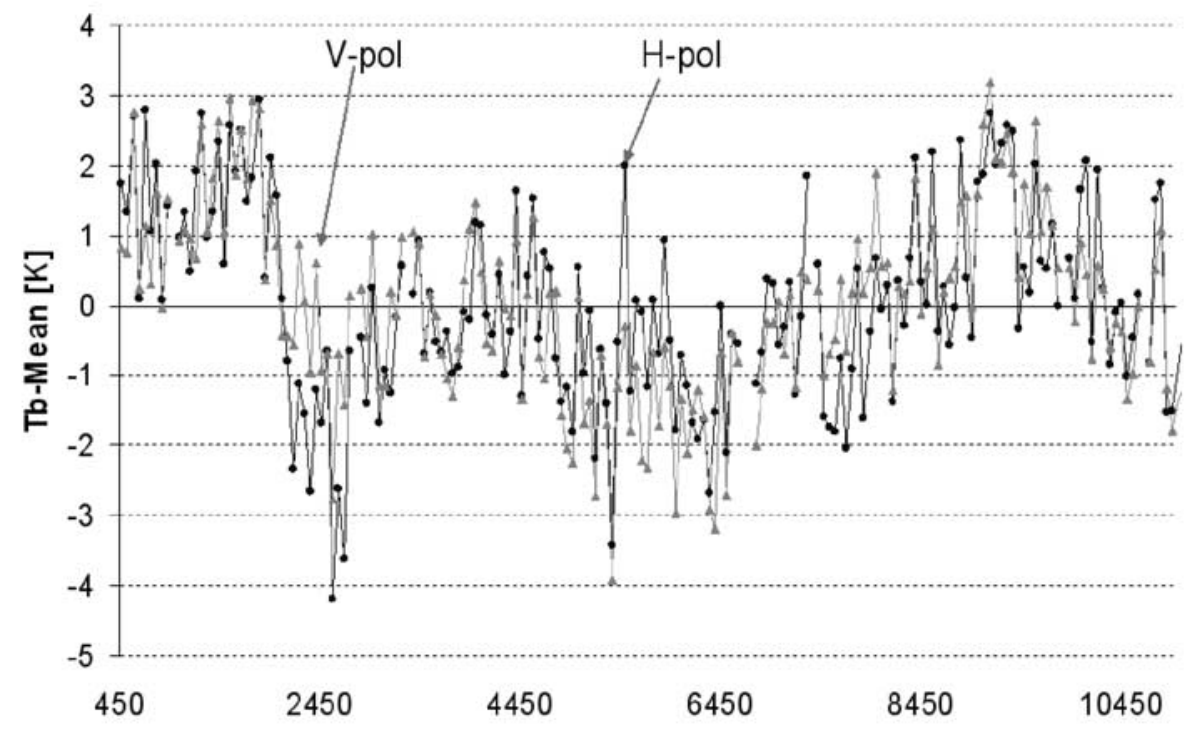

Jul. 1999 Jan. 2000

May. $2000^{\text {Rev. \# }}$ Oct. 2000

Feb. 2001 Aug. 2001

Figure 10. Pacific Ocean brightness temperature deviation from the mean. Measurements are for repeating ground swaths, approximately four days separation.

vapor as shown in Figure 5. To derive this spectral ratio, over 72,000 ocean $\mathrm{T}_{\mathrm{b}}$ points were simulated at each 10.9, 13.4 and $19.4 \mathrm{GHz}$ using atmospheric and oceanic environmental parameters from SSMI F-13 and NOAA NCEP numerical weather analysis. The spectral ratio was then calculated at each $T_{b}$ location and binned and averaged in $2 \mathrm{~mm}$ water vapor bins represented by circles. The error bars denote \pm one standard deviation. The natural logarithm of the spectral ratio was then regressed against water vapor using a third order polynomial fit shown by the solid line. Thus an estimate of the columnar water vapor, derived from collocated TMI retrievals, is used to select the proper value for the spectral ratio.

[13] Further, because the orbital measurement swaths for QRad and TMI are not collocated simultaneously, transient rain events are present in both ocean data sets that can produce significant differences (10's of Kelvin) at a given locations. This "error" is effectively removed by editing the data using TMI (and QRad) rain flags. If either instrument indicates rain, the location is deleted.

[14] For land, the emissivity is more complex, and the radiation transfer model was not used to produce equivalent $\mathrm{QRad} \mathrm{T}_{\mathrm{b}}$ 's. However, the Amazon rain forest was used because it is a large isotropic and nearly homogeneous target that is an approximate blackbody with a brightness of about $285 \mathrm{~K}$ over this range of frequencies. Small diurnal effects of a few $\mathrm{K}$ have been observed in SSM/I measurements during ascending and descending pass times that are separated by approximately 12 hours, but during the 3-day average QRad Amazon comparisons, the TMI measured brightness temperatures at 10.7 and $19.4 \mathrm{GHz}$ were averaged and linearly interpolated to compare with $\mathrm{QRad} \mathrm{T}_{\mathrm{b}}$ 's.

[15] An example of the linear regression scatter diagrams for QRad and TMI equivalent $T_{b}$ 's is given in Figure 6 for both $\mathrm{H}$ - and V-pols; and an expanded view of the difference between QRad and TMI measurements is shown in Figure 7. The symbols are binned average data on the TMI $\mathrm{T}_{\mathrm{b}}$; and the error bars denote \pm one standard deviation. The stability of this external calibration procedure is good as observed from the resulting regression slope and offset for several different calibrations during 1999 to 2003 that are provided in Table 2.

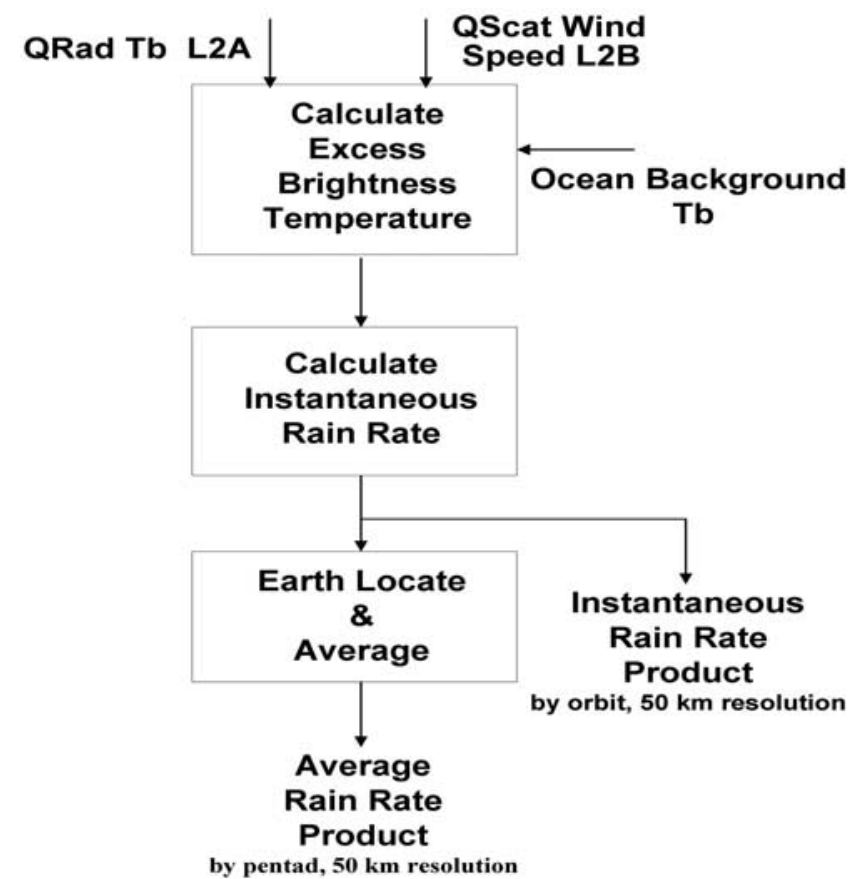

Figure 11. QRad rain rate algorithm block diagram. 


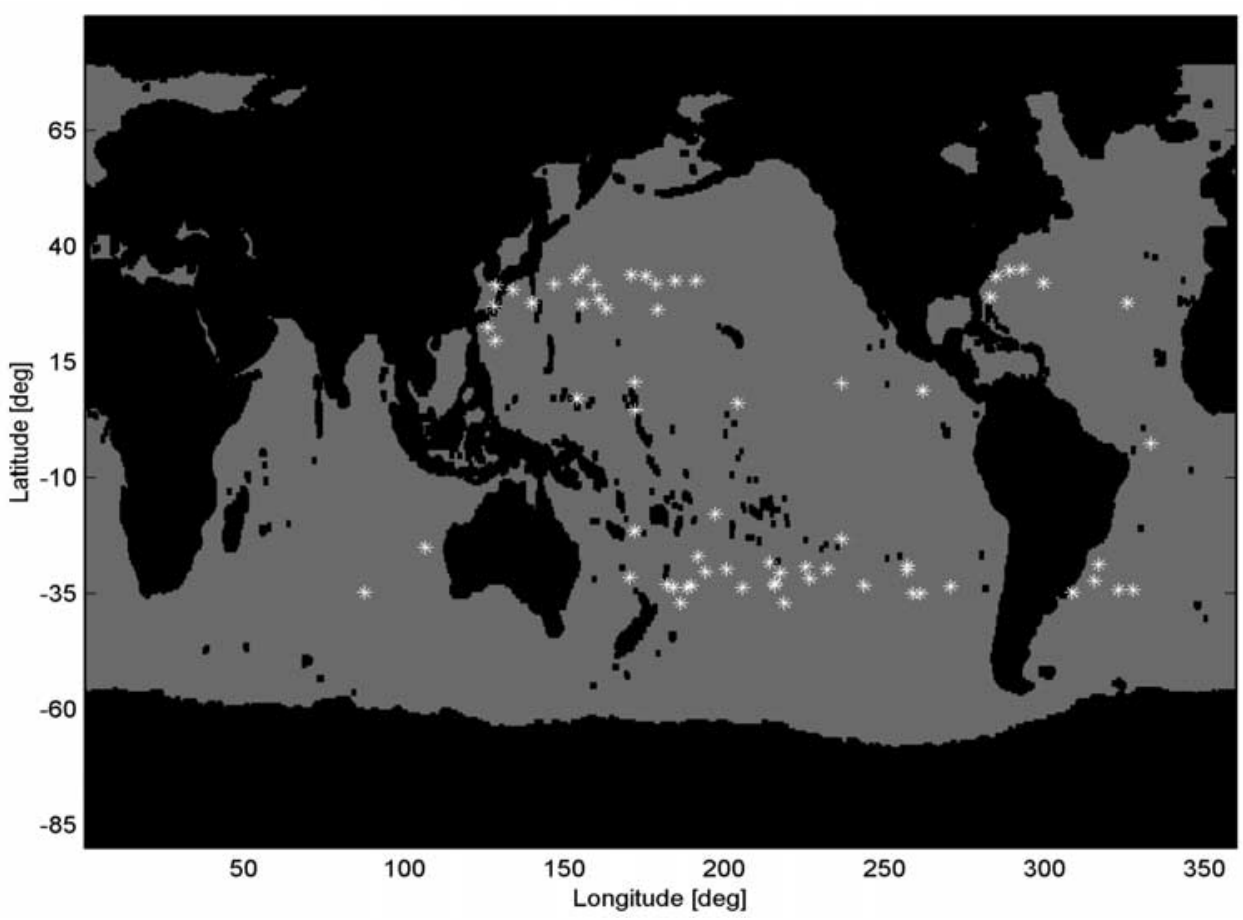

Figure 12. Collocated simultaneous rain event locations for sixty-six QRad/TMI training cases.

[16] Another assessment of the calibration stability compares histograms of QRad and TMI equivalent ocean $\mathrm{T}_{\mathrm{b}}$ 's taken seasonally. Here, three-day sets of average ocean brightness temperatures were produced with rain removed, and a typical set of histograms is shown in Figure 8. For Hpol, the QRad median $\mathrm{T}_{\mathrm{b}}$ is within a Kelvin of TMI; but for V-pol, the QRad results are low by a few Kelvin. Also QRad histograms are broader as the result of the increased QRad $\Delta \mathrm{T}$. The year 2000 calibration statistics are tabulated in Table 3; and when taken over the year, the median differences show a slight systematic variation, which may be related to the QuikSCAT seasonal thermal environment. Over a period of one year, the global mean of this variation is $-0.29 \mathrm{~K}$ with a standard deviation of 0.85 for horizontal and correspondingly $-2.76 \mathrm{~K}$ with a standard deviation of 0.75 for vertical. Again these results demonstrate that QRad and TMI derived equivalent $T_{b}$ agree on average to within $a$ few Kelvin.

[17] The final example of relative $T_{b}$ stability is shown in the approximately two-year $\mathrm{T}_{\mathrm{b}}$ time series given in Figure 9. The object of this comparison is to assess whether or not there are variable $T_{b}$ biases caused by the seasonal solar heating of the satellite and instrument. This is important because the QRad transfer function uses the physical temperature of the front-end losses to calculate $\mathrm{T}_{\mathrm{b}}$. For this evaluation, the polarized brightness temperatures are averaged over all pixels for a repeating (every 4-day) ground swath in the middle of the Pacific ocean between $\pm 45^{\circ}$ latitude. During this evaluation, it was discovered that this orbit average $T_{b}$ is very stable even when rain pixels are included. Because both earth hemispheres ( \pm latitudes) are included, the seasonal rain effects appear to cancel and the mean $\mathrm{T}_{\mathrm{b}}$ is very stable. In late 1999 , a small step in $\mathrm{T}_{\mathrm{b}}$ is visible, which corresponds to a change in the QRad range gate width (equivalent to integration time); but since then there have been no changes in the instrument transfer function. In Figure 10, the QRad average polarized $\mathrm{T}_{\mathrm{b}}$ deviation from its polarized time series mean is displayed for these repeating ground tracks, and over this two-year period, the rms difference about the mean is $1.4 \mathrm{~K}$ for both polarizations. It is encouraging that both polarized brightness temperature deviations overlay and that they are consistent with the previous analysis presented above, which shows a small seasonal variation. These results demonstrate the stability and effectiveness of this external calibration technique used for QRad; and in fact, this external TMI calibration has produced very consistent results over the entire six years that QRad has been in operation.

[18] Thus, based on analyses by Mehershahi [2000] and Jones et al. [2000], the absolute accuracy of the QRad Tb's is estimated to be better than $4 \mathrm{~K}$. While this does introduce a systematic bias error, its effects on rain retrieval are removed during algorithm training described below.

\section{Integrated Rain Rate Algorithm}

[19] The QRad rain rate algorithm is a statistical based retrieval that uses an empirical brightness temperature - rain

Table 4. Regression Coefficients for Empirical Brightness Temperature - Wind Speed Relationship

\begin{tabular}{lcccc}
\hline & $\mathrm{a}_{0}$ & $\mathrm{a}_{1}$ & $\mathrm{a}_{2}$ & $\mathrm{a}_{3}$ \\
\hline H-pol & 0 & $8.042 \mathrm{e}-2$ & $2.607 \mathrm{e}-2$ & $3.95 \mathrm{e}-4$ \\
V-pol & 0 & $1.137 \mathrm{e}-1$ & $-4.74 \mathrm{e}-4$ & $7.11 \mathrm{e}-4$ \\
\hline
\end{tabular}



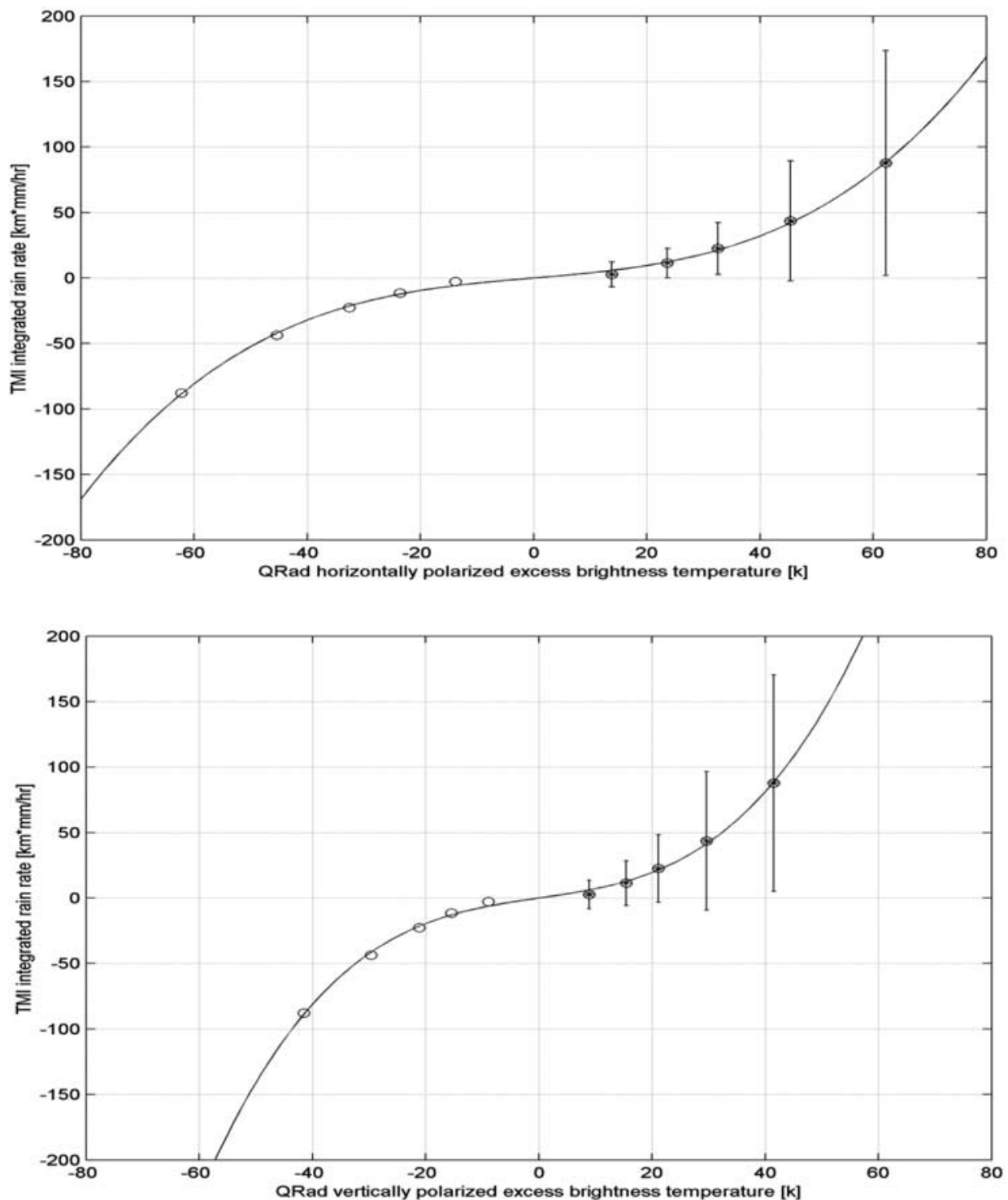

Figure 13. QRad ( $T_{e x}-R$ ) third-order transfer function for (top) H- pol and for (bottom) V- pol. Error bars denote \pm one standard deviation.

rate $\left(T_{b}-R\right)$ relationship to derive the integrated rain rate over the oceans [Ahmad et al., 2003]. Because the measured ocean brightness temperature is directly proportional to the path integrated rain rate, this is the chosen retrieved geophysical parameter. To calculate the average rain rate measured in $\mathrm{mm} / \mathrm{hr}$ requires knowledge of the rain path length. Users may convert $\mathrm{QRad}$ integrated rain rate to surface rain rate by dividing by this rain path length that is equal to the height of the rain times secant $\left(52.8^{\circ}\right)$. The QRad $T_{b}-R$ relationship was derived using a data set from rain events that were near-simultaneously observed by QRad and the TMI.

[20] A simplified algorithm block diagram is presented in Figure 11. The data inputs are (1) the QRad $\mathrm{T}_{\mathrm{b}}$ 's from the QSCAT level 2A (L2A) and the retrieved wind speed from the QSCAT level 2B (L2B) data products available at http:// podaac.jpl.nasa.gov/quikscat/ and (2) a priori information in the form of monthly-tabulated ocean background brightness temperatures.

[21] The individual polarized L2A QRad $\mathrm{T}_{\mathrm{b}}$ 's and the L2B retrieved wind speed products are provided on a spacecraft measurement grid of wind vector cells at $25 \mathrm{~km}$ resolution. These two products are earth gridded and spatially averaged to $50 \mathrm{~km}$ resolution and used with the ocean background to calculate the excess brightness upon which the rain retrieval is based. The algorithm outputs two products, namely; an earth-located instantaneous rain rate by orbit revolution at $50-\mathrm{km}$ resolution; and a five-day (pentad) rain rate average on a $0.5^{\circ} \times 0.5^{\circ}$ latitude/longitude grid. Both products are binned in 0.5 hour universal time windows. Next, the further details of the QRad rain algorithm will be presented. 
Table 5. Integrated Rain Rate - Excess Brightness Temperature Regression Coefficients and Their Respective Standard Errors Given in Parenthesis

\begin{tabular}{ccccc}
\hline & $\mathrm{b}_{0}$ & $\mathrm{~b}_{1}$ & $\mathrm{~b}_{2}$ & $\mathrm{~b}_{3}$ \\
\hline H-pol & 0 & $3.67 \mathrm{e}-1(5.67 \mathrm{e}-2)$ & 0 & $2.73 \mathrm{e}-4(1.91 \mathrm{e}-5)$ \\
V-pol & 0 & $6.26 \mathrm{e}-1(1.07 \mathrm{e}-2)$ & 0 & $8.77 \mathrm{e}-4(7.84 \mathrm{e}-5)$ \\
\hline
\end{tabular}

\subsection{TRMM Training Data Products}

[22] The QRad rain rate algorithm was trained using a data set of sixty-six significant rain events that were observed within $\pm 0.5 \mathrm{hrs}$ with TMI. Figure 12 presents the locations of these sixty-six rain events that occurred over a nine month period in 1999 and 2000. In this algorithm development activity, we use the following TRMM products available through the TRMM Science Data and Information System (TSDIS) (http://tsdis.gsfc. nasa.gov): (1) $2 \mathrm{~A} 12$ product, TMI derived surface rain rate over oceans, and (2) 3A11 product, TMI derived monthly freezing level over oceans.

[23] We use the TRMM 2A12 product to provide surface rainfall rate to train the QRad rain algorithm. The 2A12 algorithm retrieves precipitation based upon nine channels of TMI brightness temperature [Kummerow et al., 1996].
This algorithm uses a Bayesian approach that utilizes cloud resolving models to generate a large database of potential hydrometeor profiles and a microwave radiative transfer model to compute the corresponding TMI channel brightness temperatures. This algorithm generates vertical hydrometeor profiles on a pixel basis. For each pixel, cloud liquid water, precipitation water, cloud ice water, precipitation ice, and the latent heating are given at 14 vertical layers. The surface rainfall and associated confidence are also computed.

[24] We use the TRMM 3A11 product to estimate the height of the rain over the ocean for use in the QRad algorithm. The TMI 3A11 algorithm [Wilheit et al., 1991] also uses the TMI brightness temperatures to infer the freezing level, which is the estimated height of $0^{\circ} \mathrm{C}$ isotherm over oceans in $5^{\circ} \times 5^{\circ}$ boxes for one month. It also produces $5^{\circ} \times 5^{\circ}$ monthly oceanic rainfall maps using TMI Level-1 brightness temperatures.

\subsection{Excess Brightness Temperature}

[25] The oceanic microwave brightness temperature when viewed through a raining atmosphere is greater than that when viewed through a clear atmosphere. Rain can be inferred from the differential (excess) part between the raining and clear ocean $T_{b}$; so the extraction of the rain signal depends directly upon the knowledge of the ocean

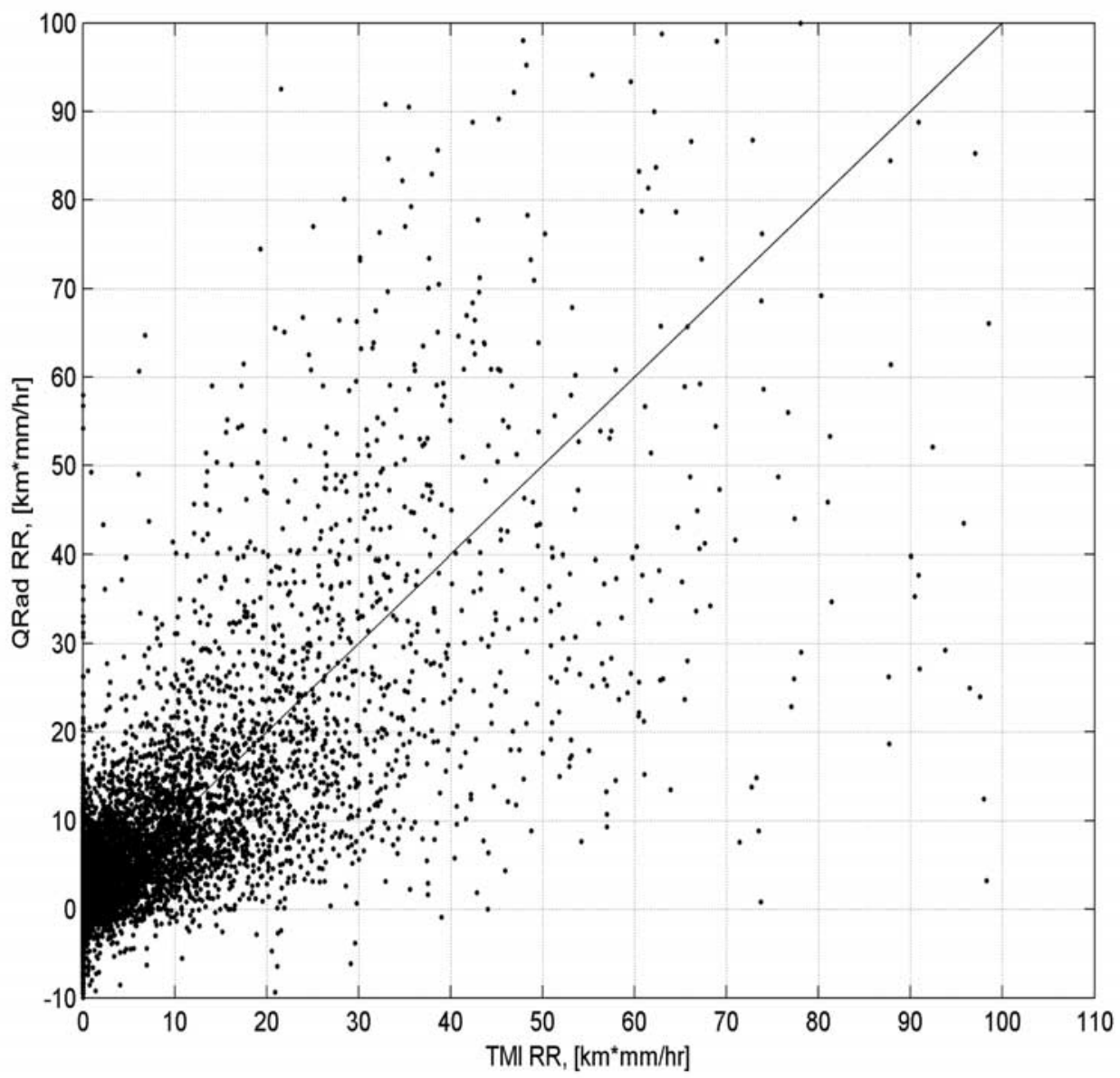

Figure 14. Instantaneous integrated rain rate comparisons for sixty-six collocated rain events for QRad and TMI. Spatial resolution is $0.5^{\circ}(50 \mathrm{~km})$. 
Table 6. Instantaneous Integrated Rain Rate Differences for Six TMI Ranges ${ }^{\mathrm{a}}$

\begin{tabular}{lcccc}
\hline TMI Range & $\begin{array}{c}\text { Number of } \\
\text { Points }\end{array}$ & $\begin{array}{c}\text { Difference } \\
\text { Mean }\end{array}$ & $\begin{array}{c}\text { Difference } \\
\text { Std. }\end{array}$ & $\begin{array}{c}\text { Difference } \\
\text { rms } /\langle\text { TMI }\rangle\end{array}$ \\
\hline $0-4$ & 2498 & 1.832 & 3.420 & 2.519 \\
$4-8$ & 984 & 0.410 & 4.896 & 0.842 \\
$8-12$ & 683 & -0.340 & 6.469 & 0.659 \\
$12-24$ & 922 & -1.983 & 10.642 & 0.636 \\
$24-32$ & 302 & -2.660 & 15.654 & 0.573 \\
$>32$ & 476 & -1.356 & 37.299 & 0.700 \\
\hline
\end{tabular}

${ }^{\mathrm{a}}$ Units are in $\mathrm{km} * \mathrm{~mm} / \mathrm{hr}$. For each range, the difference (QRad minus TMI) is calculated.

brightness when viewed through an intervening atmosphere without rain. The brightness temperature observed by the satellite microwave radiometer is determined by the electromagnetic frequency, polarization, incidence angle and by a number of atmospheric geophysical variable profiles including temperature, oxygen density, water density (vapor, cloud liquid and rain) as well as the ocean surface geophysical variables: sea surface temperature, salinity and surface wind speed. The usual remote sensing scenario is for the observing microwave radiometer to have the number of independent measurements greater than the number of unknown geophysical parameters. For example, according to Wentz and Spencer [1998], they use 7 SSM/I channels to retrieve 4 parameters; surface wind speed, integrated water vapor, integrated cloud liquid water and path average rain rate. Parameters that contribute significantly to the brightness but are not retrieved are known a priori, frequently from climatology or numerical models.

[26] Mears et al. [2000] have characterized the monthly mean ocean $\mathrm{T}_{\mathrm{b}}$ for the QRad channels using seven years of measurements from the SSM/I. This ocean brightness temperature climatology accounts for all of the geophysical parameters except the transient effects of rain and surface winds (which have been removed in the data analysis). Fortunately, the Ku-band (13.4 GHz) $\mathrm{T}_{\mathrm{b}}$ responds weakly to the atmospheric and surface geophysical parameters included in this climatological background. Further, all of these parameters vary slowly in space and time (seasonally). As an example, the dynamic range of the horizontally polarized ocean background temperature with latitude for the month of March is ( $91 \mathrm{~K} \sim 103 \mathrm{~K})$, while the vertically polarized ocean background temperature for the same month lies in the range $(165 \mathrm{~K} \sim 182 \mathrm{~K})$. In both cases, the longitude variations are almost flat.

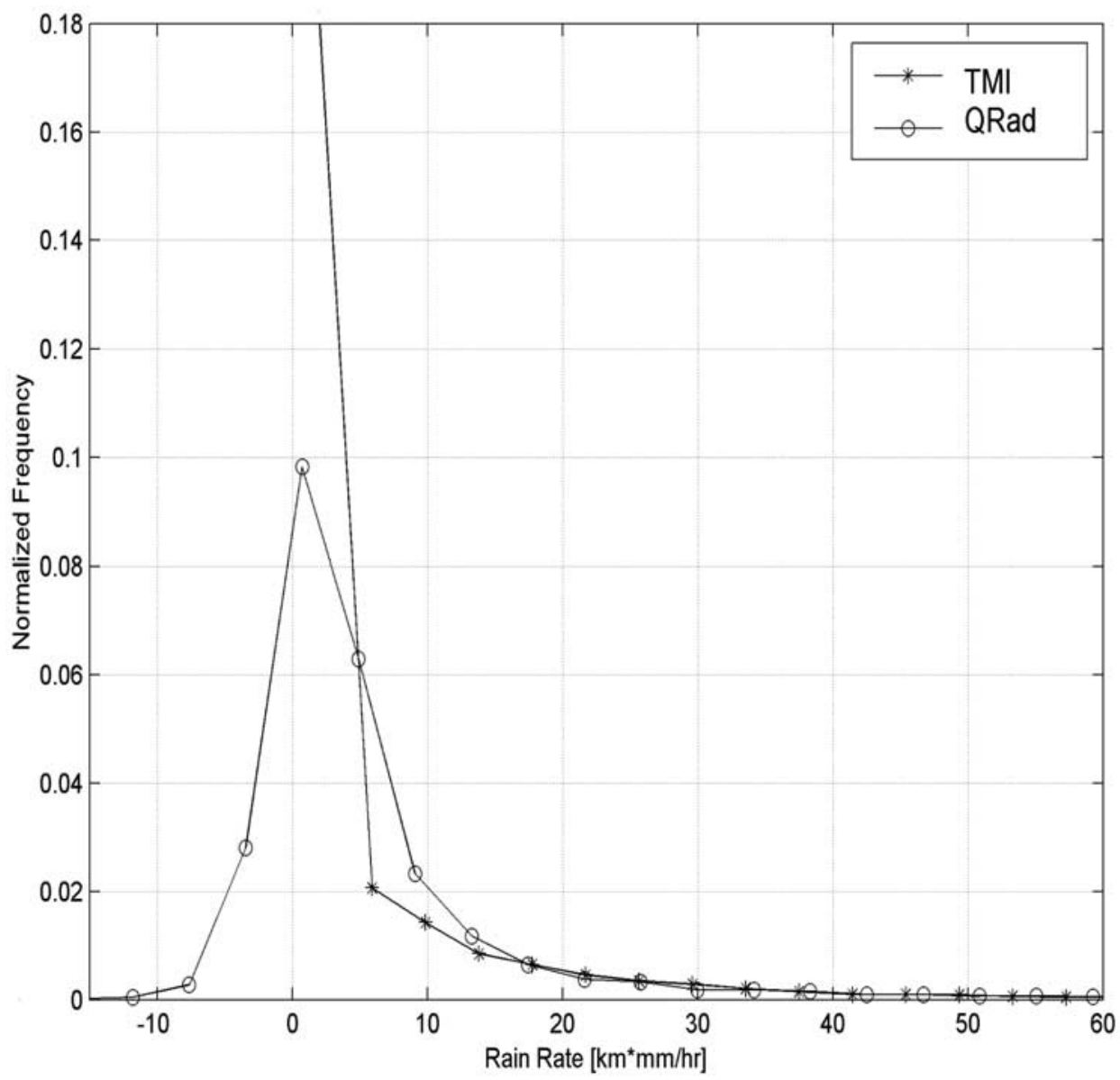

Figure 15. Probability density function for integrated rain rate at sixty-six collocated rain events for QRad and TMI. 


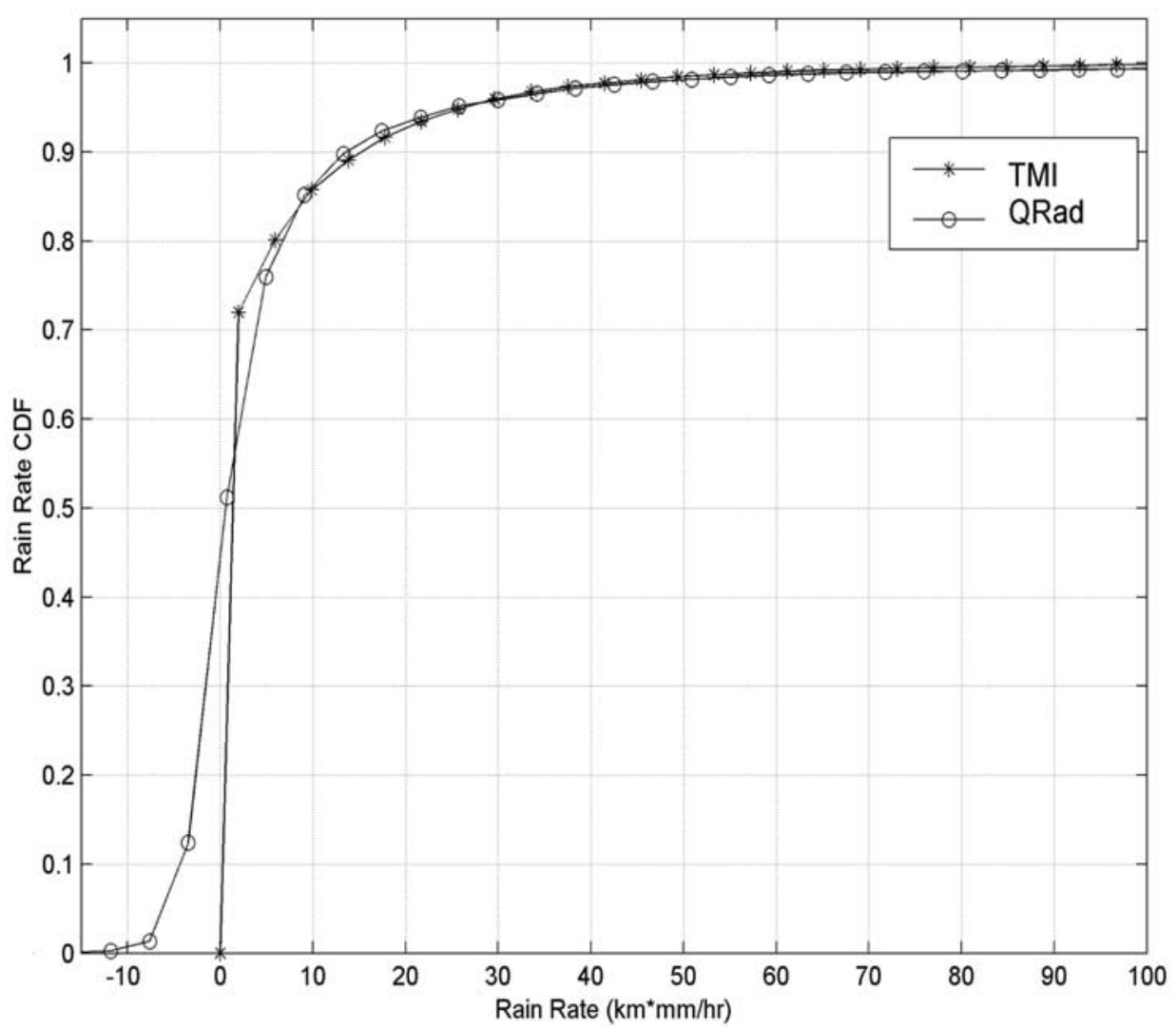

Figure 16. Cumulative distribution function for integrated rain rate at sixty-six collocated rain events for QRad and TMI.

[27] On the other hand, rain and wind are very transient with weather systems, and they need to be retrieved simultaneously. Because there are only two QRad channels (V- and H-pol), we use the ocean (and atmosphere) brightness climatology as a priori information with collocated wind speed measurements provided by the SeaWinds [QuikSCAT, 2001]. We define the "excess brightness" $\left(\mathrm{T}_{\mathrm{ex}}\right)$ as the residual of the average measured QRad $\mathrm{T}_{\mathrm{b}}$ after subtracting ocean background brightness temperature (which includes non-raining atmosphere) and the brightness temperature due to the surface wind speed. Thus the polarized $\mathrm{T}_{\mathrm{ex}}$ is

$$
\operatorname{Tex} x_{p}=T_{b_{Q R a d}-p}-T_{b_{o c e a n-p}}-T_{b_{w s p e e d} p}
$$

where

$$
T_{b Q R a d}=\frac{1}{n} \sum_{i=1}^{n} T_{b i}, \text { is the average measured QRad } \mathrm{T}_{\mathrm{b}}, \mathrm{K},
$$

where $\mathrm{n}$ is the number of pulses within a gridded measurement, $\mathrm{T}_{\text {bocean }}$ is the ocean background $\mathrm{T}_{\mathrm{b}}, \mathrm{K}$ (includes atmosphere without rain), $T_{\text {bw.speed }}$ is the $T_{b}$ due to the wind speed, $\mathrm{K}$, and $\mathrm{p}$ is the polarization.
[28] The ocean background is interpolated to the day of the observation using monthly latitude/longitude tables at $0.5^{\circ}$ spatial resolution. The QSCAT L2B ocean surface wind speeds (JPL Physical Oceanography Distributed Active Archive Center (PO.DAAC) web site: http:// podaac.jpl.nasa.gov/quikscat/) are derived from the same receiver measurements, as the $\mathrm{QRad} \mathrm{T}_{\mathrm{b}}$ measurements, and therefore they are perfectly collocated. The theoretical brightness temperature due to wind speed [Wang, 2001] is calculated as:

$$
\left(T_{b w . s p e e d}\right)_{p}=a_{0 p}+a_{1 p}{ }^{*} w s p d+a_{2 p}{ }^{*} w s p d^{2}+a_{3 p}{ }^{*} w s p d^{3}, \mathrm{~K}
$$

where $a_{i p}$ are empirical wind speed coefficients $(p=V$-pol and H-pol) given in Table 4 and wspd is the QSCAT collocated ocean surface wind speed, $\mathrm{m} / \mathrm{s}$.

[29] Typical values for the polarized brightness temperature $\left(\mathrm{T}_{\mathrm{bw} . \text { speed }}\right)$ due to a wind speed measurement of $8 \mathrm{~m} / \mathrm{s}$ are $2.5 \mathrm{~K}$ for the $\mathrm{H}$-pol and $1.2 \mathrm{~K}$ for the $\mathrm{V}$-pol. However, for ocean winds between $0-10 \mathrm{~m} / \mathrm{s}$ and in the presence of rain, QSCAT wind retrievals are typically $10-15 \mathrm{~m} / \mathrm{s}$ independent of the true wind speed. Thus, when rain is present, an erroneous wind speed correction is made, which biases the excess brightness temperature low $(\sim 5-10 \mathrm{~K})$. Fortunately, this $\mathrm{T}_{\mathrm{b}}$ error is compensated during the development of the empirical $T_{b}-R$ relationship. 


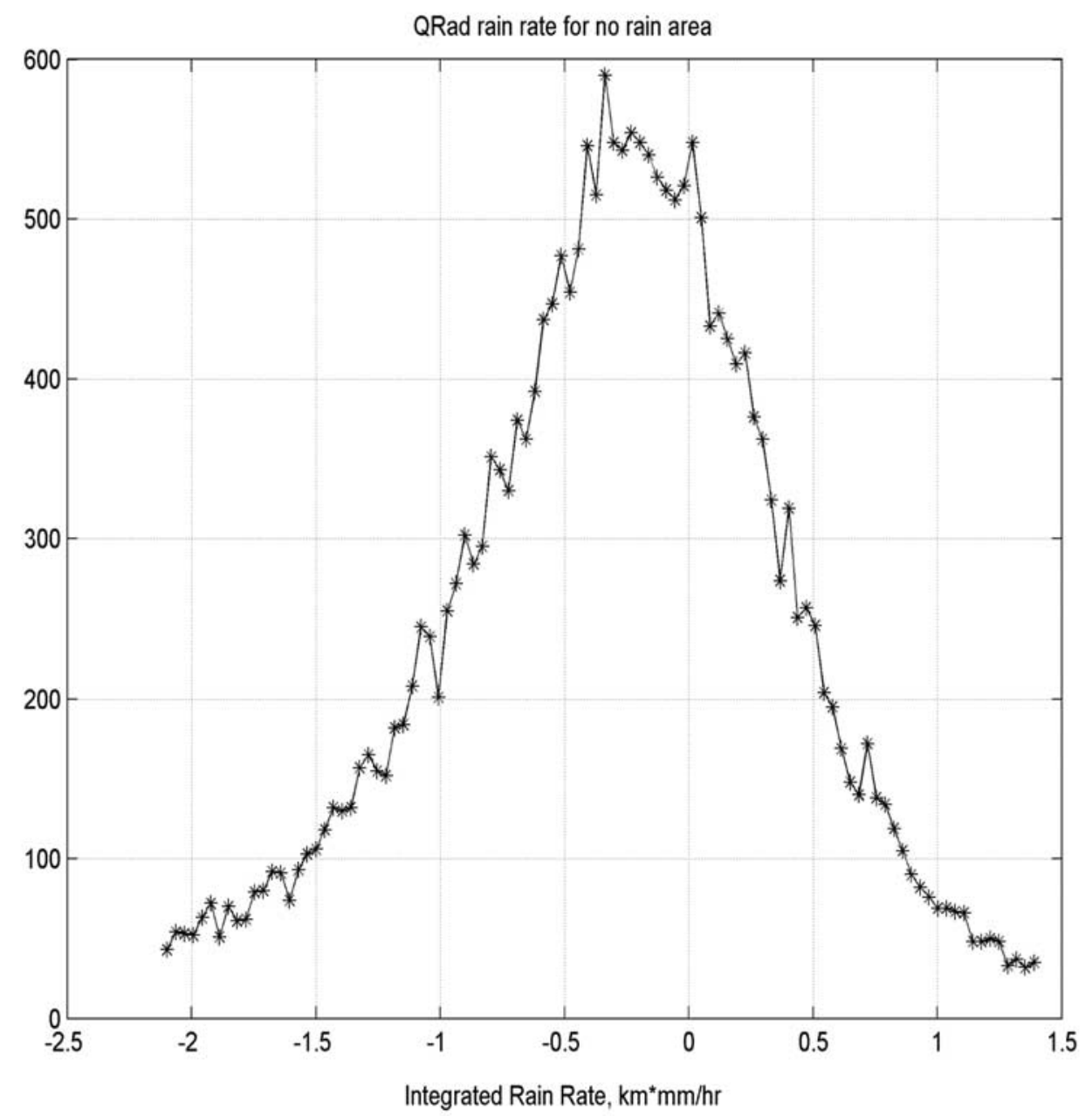

Figure 17. Integrated rain rate distribution for rain-free regions for QRad instantaneous rain product at $0.5^{\circ}(50 \mathrm{~km})$ spatial resolution.

\subsection{Excess Brightness - Integrated Rain Rate Relationship}

[30] The rain rate algorithm is a statistical based retrieval that uses an empirical brightness temperature - rain rate $\left(T_{b}-R\right)$ relationship. This relationship is derived using a QRad brightness temperature and TMI integrated rain rate data set from sixty-six significant rain events that are observed within $\pm 0.5 \mathrm{hrs}$. In the propagation direction, the total atmospheric absorption and emission of microwave energy is directly proportional to the rain path length; thus the observed rain brightness temperature is proportional to the integrated rain rate.

[31] The $T_{b}-R$ relationship is calculated using a regression analysis of the QRad excess brightness $\left(\mathrm{T}_{\mathrm{ex}}\right)$ with the corresponding collocated TMI integrated rain rate (IRR). First QRad $\mathrm{T}_{\mathrm{ex}}$ are produced on $0.25^{\circ}$ grid, and the corresponding TMI 2A12 surface rain rates are converted to IRR. Because the TMI integrated rain rate value is not available in $2 \mathrm{~A} 12$, the IRR is approximated to be the product of the TMI surface rain rate $(\mathrm{mm} / \mathrm{hr})$ and the rain path length $(\mathrm{km})$. For this calculation, we use the TMI retrieved freezing level (TMI 3A11 product) as the rain height interpolated to $0.5^{\circ}$ spatial resolution and multiply by the secant of the TMI incident angle $\left(52.8^{\circ}\right)$. For example, a typical average value for rain height near the equator during the month of March 2000 is about $4.9 \mathrm{~km}$.

[32] Next, the $T_{\text {ex }}$ and the IRR are averaged over a $0.5^{\circ} \times$ $0.5^{\circ}$ earth grid that corresponds to the effective resolution of the QRad antenna. In this manner, we transfer the beam-fill correction from TMI to QRad. At high rain rates associated with small convective rain cells, the beam-fill correction does not scale well, thus QRad rain rates will be significantly under estimated. Finally, these data are binned by TMI IRR, averaged and then used in a least-squares curve fit procedure to determine an optimal 3rd order polynomial. This polynomial is forced to pass through the origin, which produces a $\mathrm{T}_{\mathrm{b}}-\mathrm{R}$ function with odd symmetry about zero $\mathrm{T}_{\mathrm{ex}}$. This odd function regression is adopted to cancel (in the mean) the effect of the QRad measurement noise $(\Delta \mathrm{T})$ that frequently causes the $T_{\text {ex }}$ to be negative at low rain rates. The estimated coefficients along with their respective estimated standard errors, are provided in Table 5, and Figure 13 shows the resulting transfer function with error bars of \pm one standard deviation for each bin. 


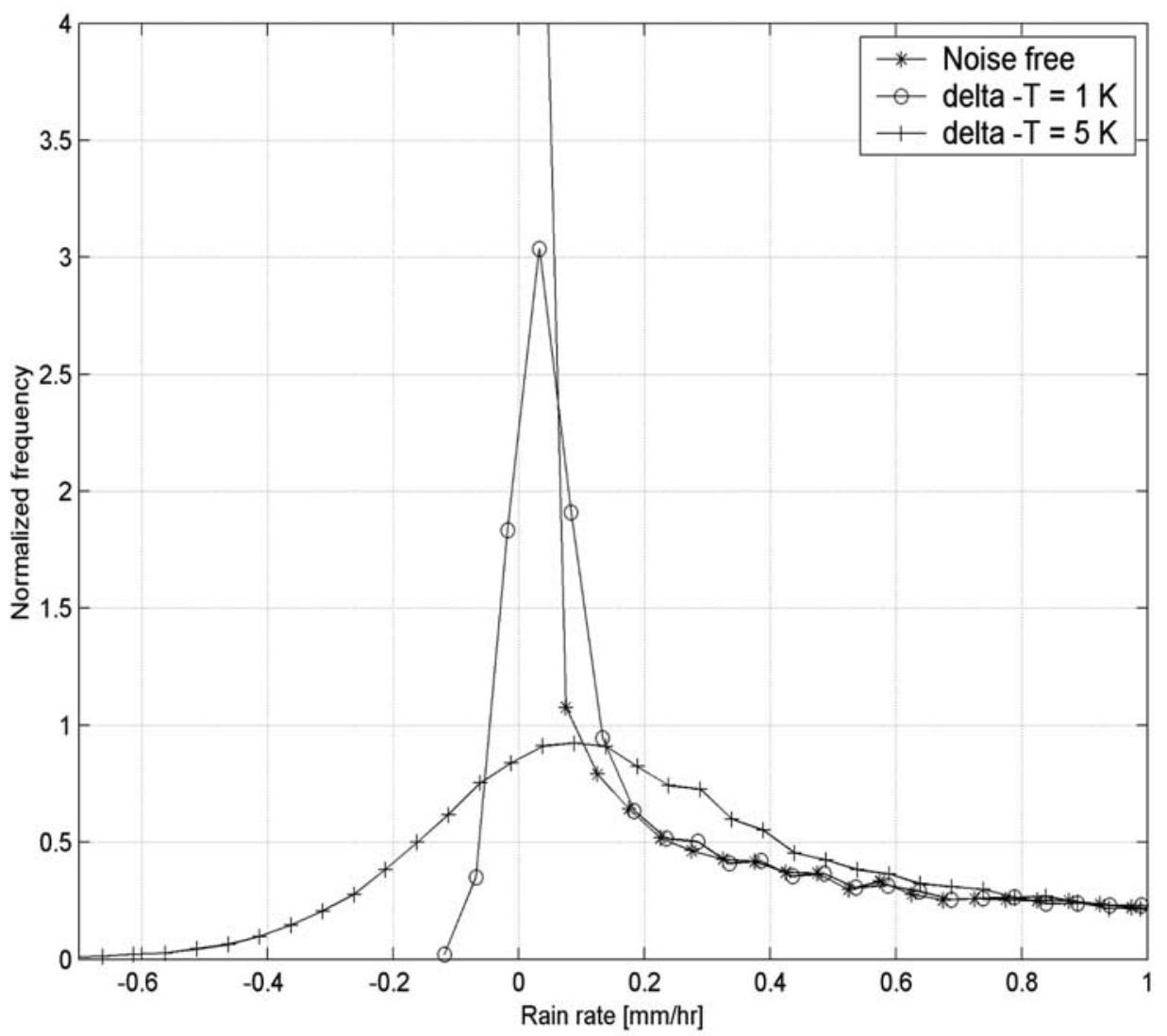

Figure 18. Retrieved rain rate histograms from Monte Carlo simulation. Three curves are for noise-free $\mathrm{Tb}$ measurement (asterisks), $\Delta \mathrm{T}=1 \mathrm{~K}$ (open circles), and $=5 \mathrm{~K}$ (pluses).

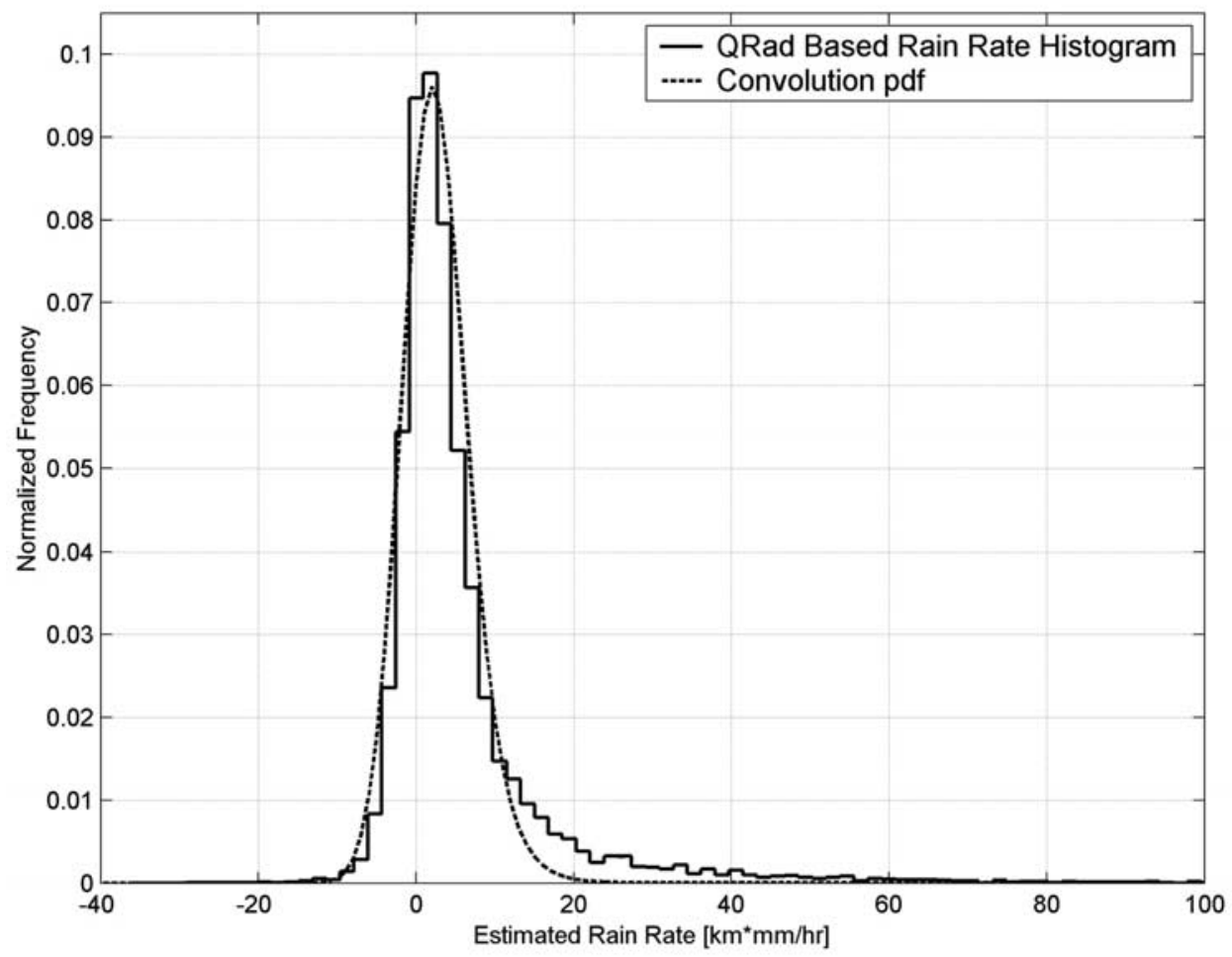

Figure 19. QRad estimated rain rate histogram and fitted convolution probability density. 


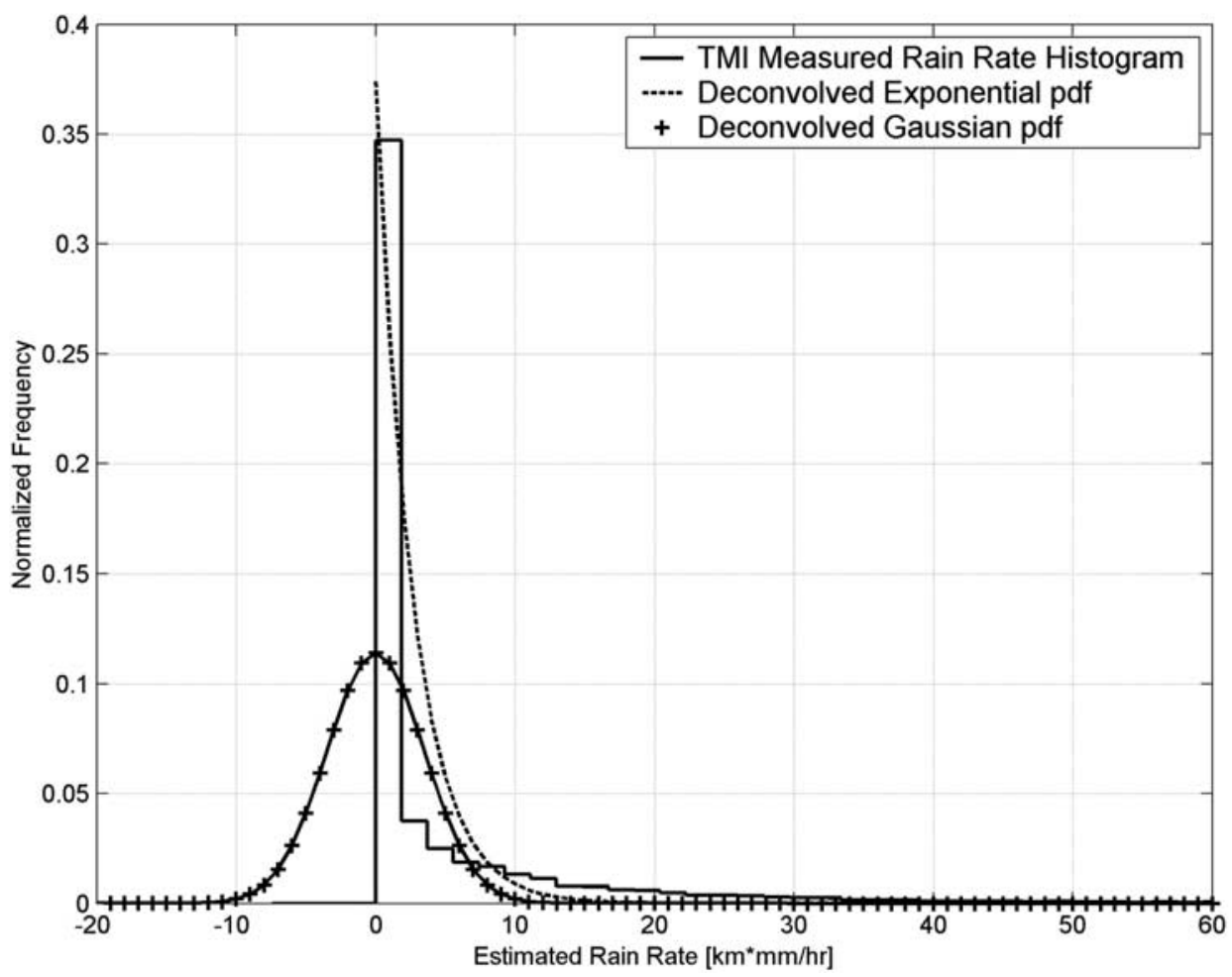

Figure 20. Deconvolution of the estimated rain rate pdf into estimated constituent Gaussian and exponential pdfs.

\subsection{Integrated Rain Rate}

[33] The integrated rain rate is calculated from the polarized $T_{e x}$ using the $T_{b}-R$ relationship given as:

$$
I R R_{p}=b_{0 p}+b_{1 p} * \operatorname{Tex}_{p}+b_{2 p} * \operatorname{Tex}_{p}^{2}+b_{3 p} * \operatorname{Tex}_{p}^{3}
$$

where $b_{i}$ is the regression coefficients, given in Table 5 .

[34] The final rain rate is the weighted-average of the polarized rain rates. The usual procedure is to weight measurements by their inverse variances; but for QRad, the variances for $\mathrm{V}$ - and $\mathrm{H}$-pol are similar. However, the dynamic range of the $T_{\text {ex }}$ 's differ by approximately a factor of two (H-pol $\sim 2 \times \mathrm{V}$-pol range); therefore we weight these two rain retrievals by their dynamic ranges. Since Hpol is less affected by the QRad $\Delta \mathrm{T}$ noise, it is given greater weight in the final result:

$$
I R R=c_{0}+c_{1} \frac{\left(2 \cdot I R R_{h}+I R R_{v}\right)}{3} k m * m m / h r
$$

where $c_{o}$ is the empirically derived bias for no rain areas and $c_{1}$ is the empirically derived slope that matches the TMI training data set rain accumulation. In the current version of the algorithm, $\mathrm{c}_{\mathrm{o}}$ and $\mathrm{c}_{1}$ have values of approximately zero and unity, respectively.

[35] A quantitative comparison between the QRad and TMI IRR's for the sixty-six rain events is presented as a scatter diagram in Figure 14. For low TMI IRR's $(<3 \mathrm{~km} * \mathrm{~mm} / \mathrm{hr})$, the QRad algorithm produces about $50 \%$ negative rain rates as a result of the $\Delta \mathrm{T}$ measurement noise; however for larger IRR's the retrievals are well behaved in the mean. This may be better examined in the statistics of differences (QRad minus TMI) presented in Table 6. For this comparison, we use the same data as Figure 14; but now we bin the data in six ranges of TMI IRR. The mean of the individual histograms is near zero, that verifies the $T_{b}-R$ least mean squares regression procedure; however the standard deviations are large as a result of the poor QRad $\Delta \mathrm{T}$.

[36] Additional quantitative comparisons between TMI and QRad for the sixty-six rain events are presented in terms of the IRR probability density functions (pdf's) and cumulative distribution functions (cdf's) shown in Figures 15 and 16, respectively. Note that in Figure 15, only rain rates greater than zero are presented for TMI. The large $\Delta \mathrm{T}$ causes some distortion in the QRad pdf especially for low IRR; however, this does not produce a significant accumulation error as seen by examining the QRad cdf. This is the result of using the $c_{1}$ coefficient in equation (7) to adjust QRad to match the TMI IRR accumulation.

[37] To evaluate the hypothesis that averaging negative and positive rain rates results in the proper mean value, we examined the histograms of QRad IRR's for 50-km pixels over large non-raining areas. For this analysis, about sixteen orbits were examined and many large regions at least $10^{\circ} \times$ $10^{\circ}$ were selected where there was apparently no rain, which resulted in about 10,000 pixels. Histograms were examined individually and collectively with similar results as presented in Figure 17. The mean IRR of these combined non-raining areas is nearly zero $(-0.355 \mathrm{~km} * \mathrm{~mm} / \mathrm{hr})$. We use this small offset in the QRad rain retrieval algorithm, 


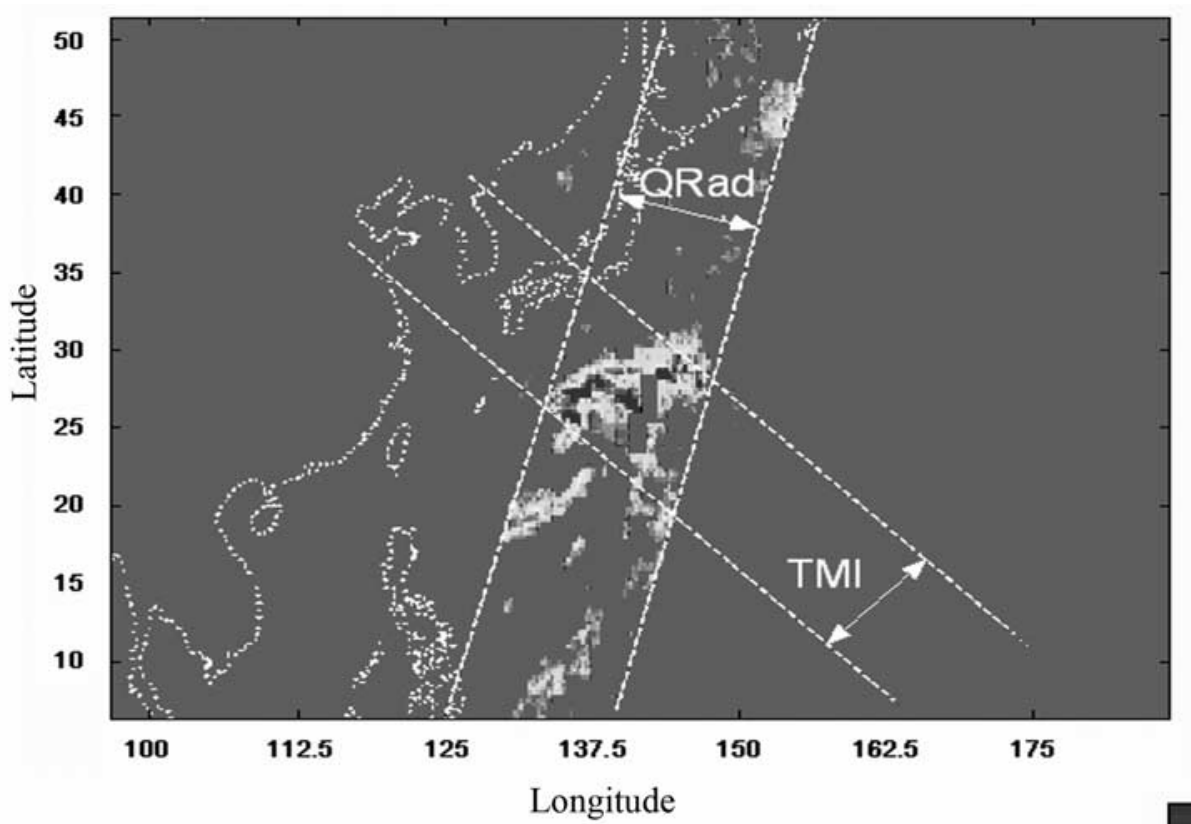

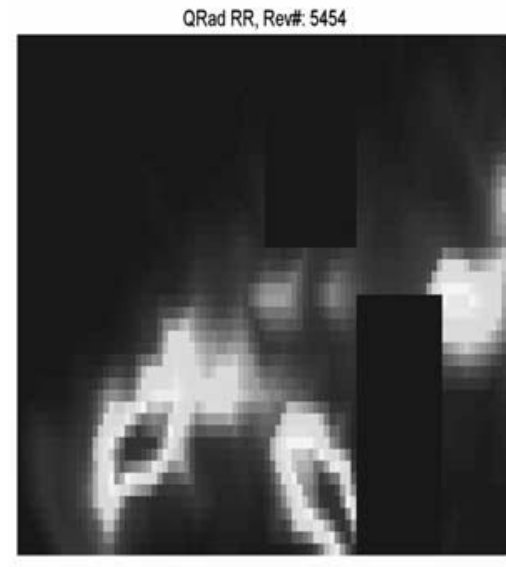

QRad

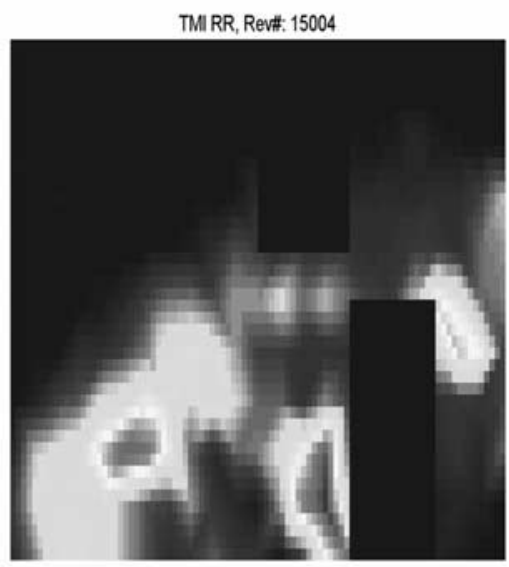

TMI

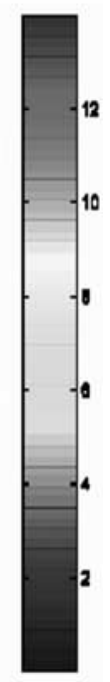

Figure 21. Example of instantaneous rain rate images produced by QRad and TMI. Spatial sampling is $0.125^{\circ}(12.5 \mathrm{~km})$, and coincidence time difference is $\sim 20 \mathrm{~min}$. See color version of this figure at back of this issue.

bias $c_{o}$ in equation (7), to make the average instantaneous rain product zero mean for non-raining areas.

[38] Since the QRad rain rate algorithm is applicable only over the ocean, we use a conservative land mask with extended land boundaries (and small islands deleted) to determine where the rain rate algorithm is applied. Unfortunately, when QRad measurements are close to land, the measured $T_{b}$ is also affected by the "hot" radiance from land that enters through the antenna pattern side-lobes. Thus, within about $150 \mathrm{~km}$ of land, the measured QRad $\mathrm{T}_{\mathrm{b}}$ has a land bias of about +5 to $+10 \mathrm{~K}$. To compensate for this effect, the background brightness temperature over the land is set to its typical value of $270 \mathrm{~K}$, and the ocean/land background is smoothed using a $3 \times 3$ pixel window to eliminate the effect of the sharp land-ocean boundary. In this way, the ocean brightness temperature near the boundary is elevated in an attempt to remove the influence of land on Tex. The final step is to evaluate the monthly rain rate at all land/water boundaries and identify anomalous negative rain rates, when ocean background is too high; and positive rain rates, when ocean background is too low. The final land mask is subjectively adjusted to remove these anomalous rain rates that may result along the land borders.

\subsection{Rain Retrieval Errors}

[39] There are several sources of error in the retrieved rain rates; but the one that predominates is the random component of the QRad brightness temperature measurement error. Because of this larger than normal $\Delta \mathrm{T}$, the excess brightness temperature includes a large random, zero-mean, Gaussian noise component that distorts the retrieved rain rate $\mathrm{pdf}$ and even produces unrealistic negative rain rates. We believe that after spatial and temporal averaging of both positive and negative rain rates, the majority of this noise will 


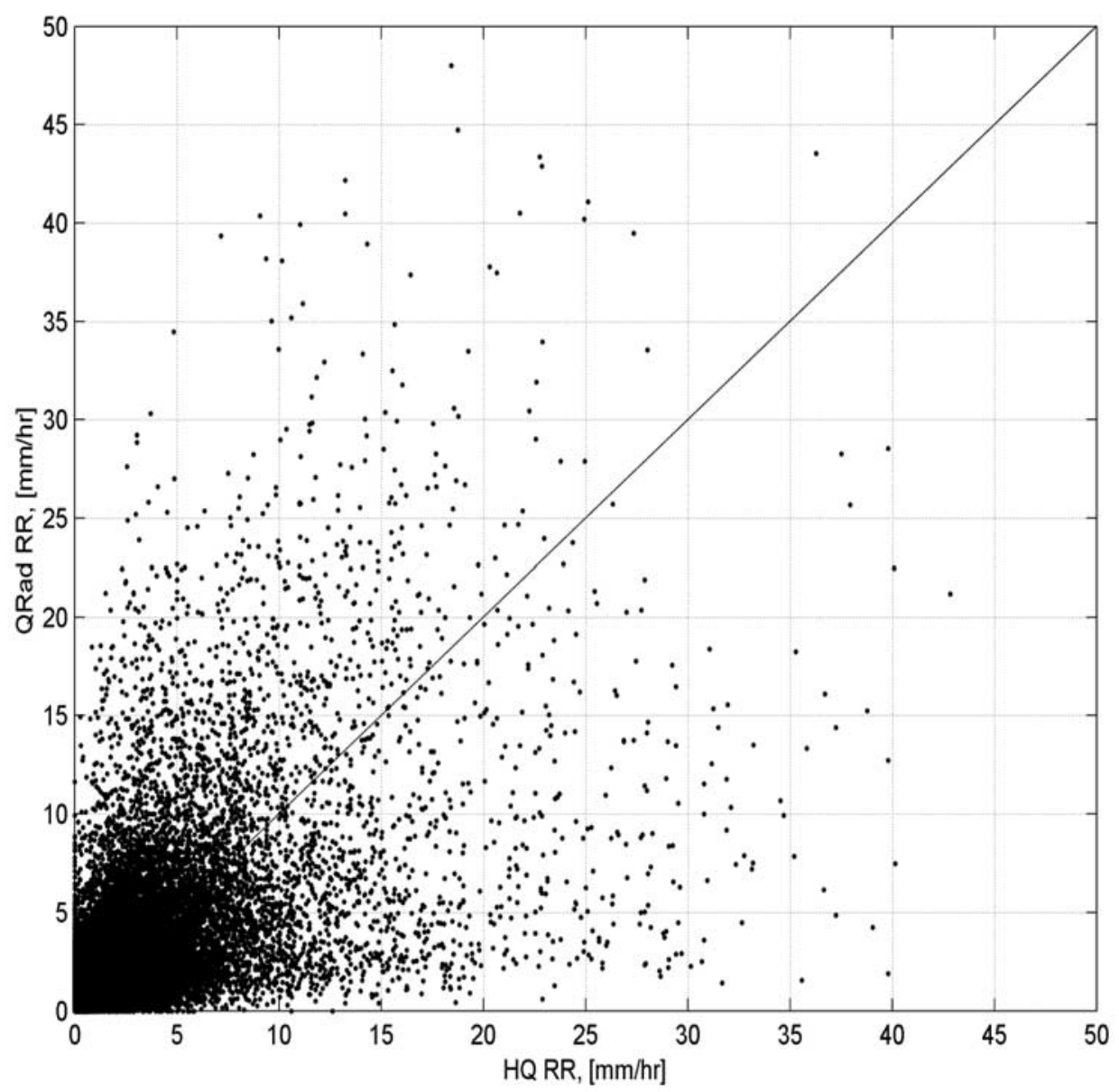

Figure 22. Instantaneous rain rate comparisons for a hundred and eight collocated rain events for QRad and TRMM 3B42RT HQ (TMI and SSM/I) product. Spatial resolution is $0.25^{\circ}(25 \mathrm{~km})$ and coincidence time difference is $<75 \mathrm{~min}$.

cancel, and the result will be a reasonable estimate of the true average rain rate. For non-raining regions, the average of the negative and positive rain rates will be zero (after a small bias is removed); and for raining regions, the average approaches the true rain rate. This is the fundamental premise of our rain rate retrieval. To assess the effect of random measurement $\Delta \mathrm{T}$ on the rain rate retrieval, convolution models are applied both in the forward and inverse directions.

[40] First, a Monte Carlo simulation was performed in the forward direction [Wang, 2001]. The TMI measured rain rate density was assumed to be "nature", and the "noisefree" excess brightness temperatures were generated using the inverse $T_{b}-R$ relationship. Next, noisy $T_{e x}$ were created by adding Gaussian noise, and then converted to rain rate using the $T_{b}-R$ relationship. Results for noise-free and noisy rain retrievals are presented in Figure 18 for $\Delta \mathrm{T}$ 's of $1 \mathrm{~K}$ and $5 \mathrm{~K}$. The $\Delta \mathrm{T}=5 \mathrm{~K}$ simulation is representative of the $\mathrm{QRad}$ $\mathrm{T}_{\mathrm{ex}}$.'s after averaging over the $0.5^{\circ} \times 0.5^{\circ}$ grid. As expected, the noisy rain retrieval density functions are the convolution of the Gaussian measurement error pdf with the noise-free rain rate density. The effect of the QRad measurement $\Delta \mathrm{T}$ is most obvious at low rain rates where the approximately exponential noise-free density function is significantly reduced in amplitude and broadened. Further, physically unrealistic negative rain rates are produced.

[41] Next, a convolution model is applied to the estimated QRad rain rates to deconvolve the measurement noise and rain rate populations. The analytical form of the convolution probability density function can be readily found from the analytical forms of the Gaussian and exponential components via the convolution integral formula. Once the overlaid convolution pdf has obtained a good fit to the empirical data, the component distributions can be resolved and

Table 7. Instantaneous Rain Rate Differences for Five TRMM 3B42RT HQ Data Ranges ${ }^{\mathrm{a}}$

\begin{tabular}{ccccc}
\hline HQ Range & $\begin{array}{c}\text { Number of } \\
\text { Points }\end{array}$ & $\begin{array}{c}\text { Difference } \\
\text { Mean }\end{array}$ & $\begin{array}{c}\text { Difference } \\
\text { Std. }\end{array}$ & $\begin{array}{c}\text { Difference } \\
\mathrm{rms} /\langle\mathrm{HQ}\rangle\end{array}$ \\
\hline $0-1$ & 14582 & 0.5330 & 0.9548 & 2.6428 \\
$1-2$ & 7842 & 0.3571 & 1.7383 & 1.2278 \\
$2-4$ & 6492 & 0.1350 & 2.7590 & 0.9736 \\
$4-8$ & 3637 & -0.5062 & 4.0268 & 0.7385 \\
$>8$ & 1939 & -4.2267 & 8.9058 & 0.7218 \\
\hline
\end{tabular}

${ }^{\mathrm{a}}$ Units are in $\mathrm{mm} / \mathrm{hr}$. For each range, the difference (QRad minus HQ) is calculated. 


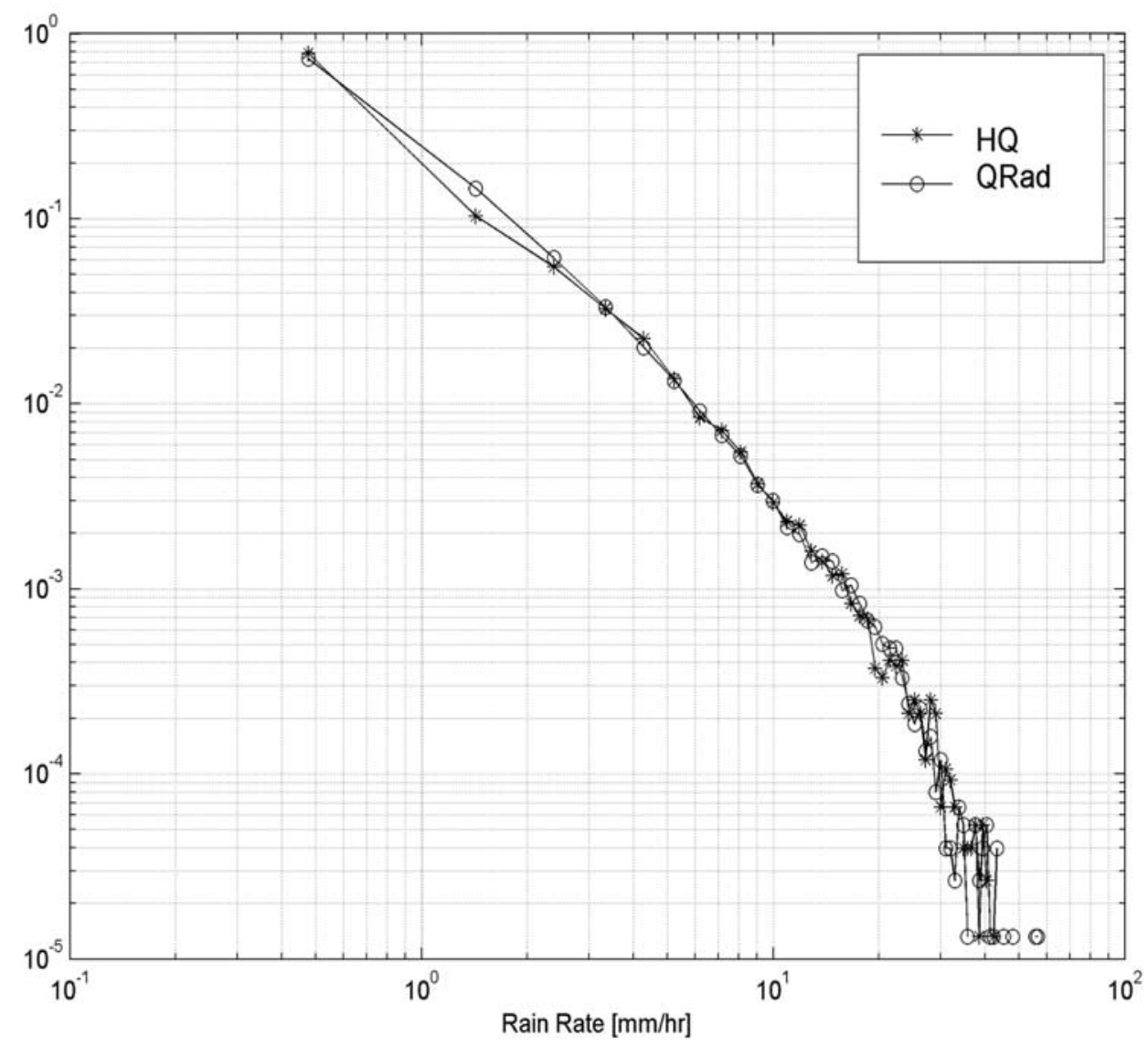

Figure 23. Rain rate probability density function for a hundred and eight collocated rain events for QRad and TRMM 3B42RT HQ (TMI and SSM/I) product.

examined. The fit is obtained simply by varying the unknown parameters of the convolution density. Note that the convolution density is parameterized by the constituent pdf parameters; specifically, the mean and variance of the Gaussian distribution and the mean (or shape parameter) of the exponential distribution. Figure 19 shows the histogram of QRad estimated rain rates developed from equation (7) as well as a candidate fitted convolution density of a Gaussian and exponential. Although the convolution model provides a good fit for small rain rates, it underestimates the proportion of data in the right tail, i.e. rain rates larger than $15 \mathrm{~km} * \mathrm{~mm} / \mathrm{hr}$ or more. Figure 20 shows the subsequent constituent distributions compared to the TMI rain rates which are taken to be "nature". Clearly, the exponential model is inadequate in that it does not roll off fast enough to fully capture the tail behavior of the rain rate distribution. However, the "smearing" effect and relative size of the QRad measurement $\Delta \mathrm{T}$ can be obtained by examination of the estimated Gaussian distribution; specifically the estimated standard deviation which was found to be $3.5 \mathrm{~km} * \mathrm{~mm} / \mathrm{hr}$ in this case. This estimate provides a quantitative way to assess the effect of the measurement $\Delta \mathrm{T}$ on the QRad rain rate estimation procedure.

[42] It is readily observed that the convolution models in both the forward and inverse directions fail to exhibit an adequate fit in the tail of the empirical distribution of estimated (QRad derived) and calibration (TMI) rain rates. However, these examples illustrate that once a sufficient pdf model can be developed to model the rain rate distribution, the convolution model does have merit in illustrating the effect of measurement $\Delta \mathrm{T}$ on the QRad estimates. Further investigation into more appropriate rain rate pdf models as well as objective parameter estimation techniques are currently being developed. Other secondary sources of error are the result of the following:

[43] 1. Convective and stratiform rain type differences. For the same rain rate, different rain types can produce differences in brightness temperatures of order a few 10's K, which is neglected in the $T_{e x}-R$ relationship. To compensate for this effect, the QRad/TMI training data set was selected over a range of geographic locations and seasons to produce an average $T_{b}-R$ relationship based upon the convective/ stratiform conditions encountered.

[44] 2. Beam-fill differences between QRad and TMI due to antenna spatial resolution. The SeaWinds scatterometer antenna was designed to optimize the antenna boresight gain at the expense of the antenna main beam efficiency. As a result, the QRad antenna collects energy over an effective surface area that is approximately twice that of the TMI (50 km sampling for QRad compared to $25 \mathrm{~km}$ sampling for $\mathrm{TMI})$. To produce the $\mathrm{QRad} \mathrm{T}_{\mathrm{b}}-\mathrm{R}$ relationship, the TMI rain rates were averaged over $50 \mathrm{~km}$ to match the $\mathrm{QRad} \mathrm{T}_{\mathrm{b}}$ 

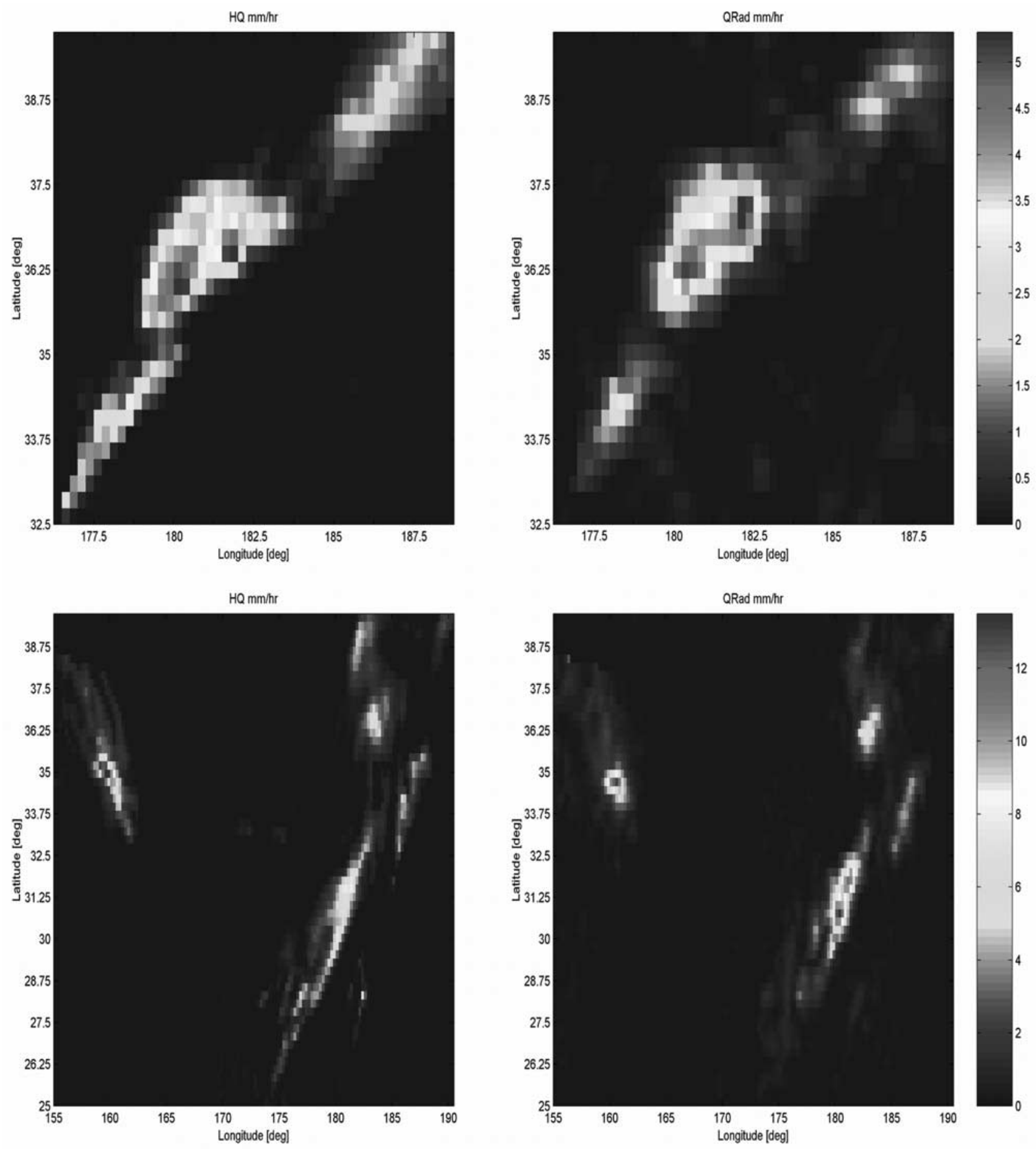

Figure 24. Examples of rain events measured by QRad (right) and TRMM 3B42RT HQ (TMI and $\mathrm{SSM} / \mathrm{I})$ product (left). Spatial resolution is $0.25^{\circ}(25 \mathrm{~km})$ and coincidence time difference $<35 \mathrm{~min}$. See color version of this figure at back of this issue.

measurement resolution; but the empirical beam filling corrections applied to TMI do not scale linearly. This will result in a systematic underestimation of QRad peak rain rates compared to the TMI measurements.

[45] 3. Long term systematic radiometric calibration drift. The QRad calibration stability illustrated in Figure 10 shows an rms variation of $1.4 \mathrm{~K}$. This effect contributes to uncertainty in the empirically derived coefficients used in the retrieval algorithm and to biases in the average retrieved rain rates. Nevertheless, this error source is considered secondary to the error introduced by the large $\mathrm{QRad} \Delta \mathrm{T}$.

[46] 4. QSCAT wind speed retrieval errors and the resulting reduction in excess brightness temperatures. In the 

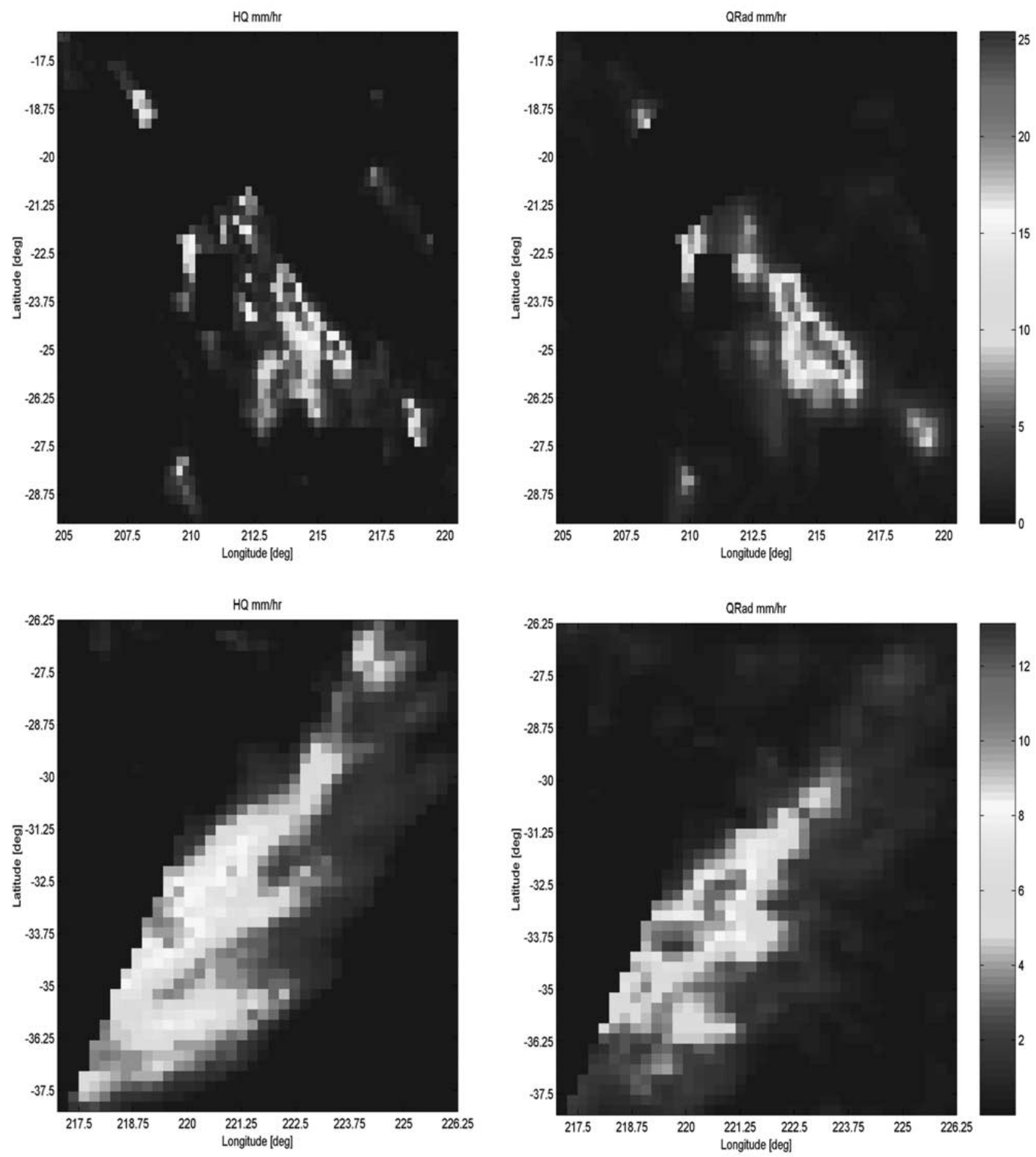

Figure 25. Other examples of rain events measured by QRad (right) and TRMM 3B42RT HQ (TMI and SSM/I) product (left). Spatial resolution is $0.25^{\circ}(25 \mathrm{~km})$ and coincidence time difference $<60$ min. See color version of this figure at back of this issue.

presence of rain (and at low to moderate ocean wind speeds), QSCAT wind retrievals are bogus, typically 10$15 \mathrm{~m} / \mathrm{s}$ independent of the true wind speed. This error can lower the $\mathrm{T}_{\mathrm{ex}}$ by $5-10 \mathrm{~K}$ (worst case); however this effect is largely compensated by the empirical $\mathrm{Tb}-\mathrm{R}$ relationship regression; thus this is not considered to be a significant source of rain retrieval error.
[47] 5. Inter-annual variability in the ocean (and atmosphere) background from climatology. Because of the low sensitivity of the $13.4 \mathrm{GHz}$ brightness to the atmospheric and oceanic physical variables, this error source is not believed to be dominant. For example, qualitative comparisons with rain patterns from TMI (presented in section-4) do not show significant differences in the global rain 

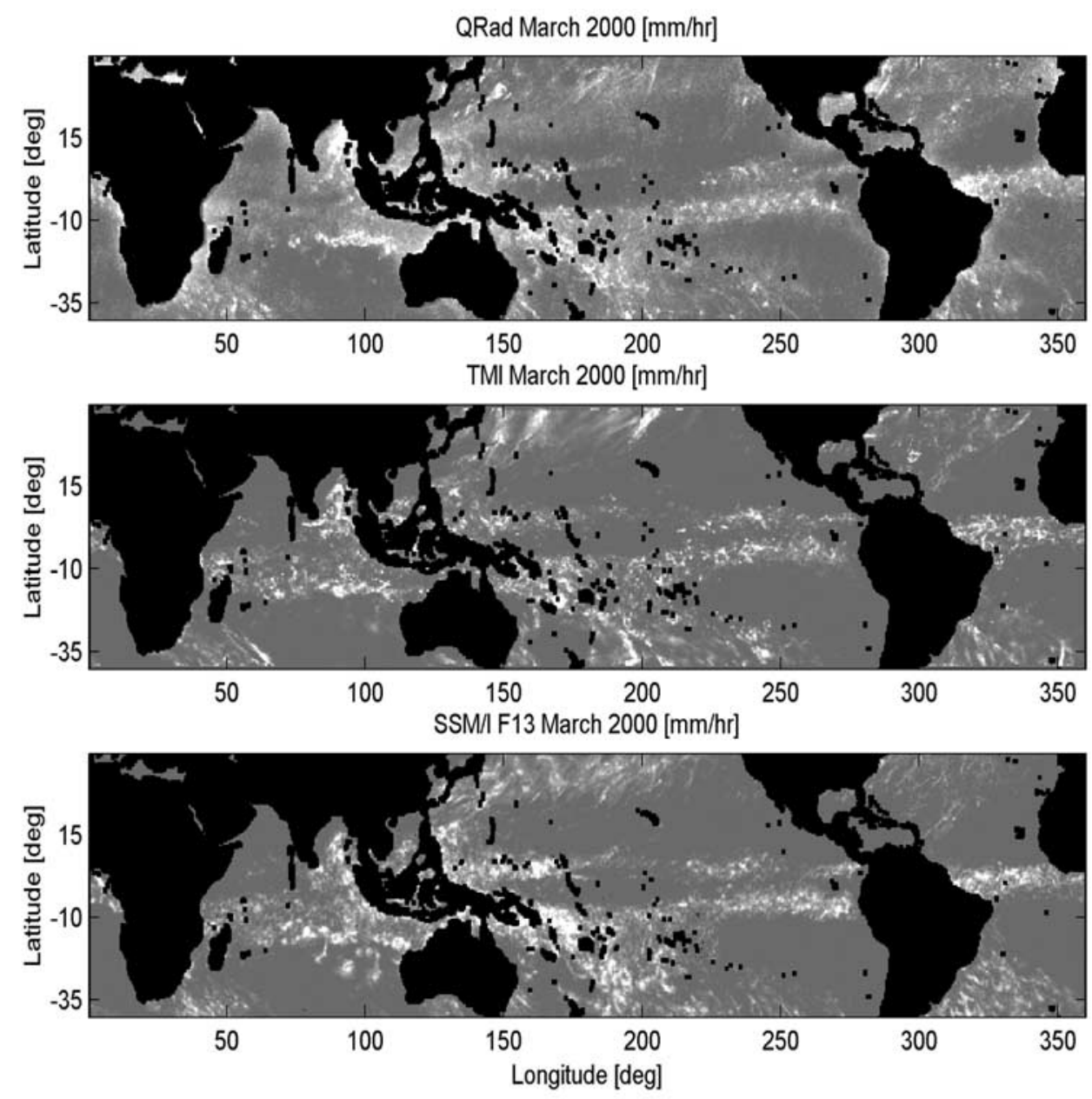

Figure 26. Monthly rain images produced by QRad, TMI and SSM/I F13 for March 2000. Spatial resolution $0.5^{\circ}(50 \mathrm{~km})$.

images. This is supported by good comparisons between the ocean background and the three-day brightness temperatures used in the QRad external $\mathrm{T}_{\mathrm{b}}$ calibration. This is significant because the QRad effective brightness derived from TMI still has transient effects of winds (rain flags remove transient effects of rain) in addition to inter-annual variability of other variables from climatology. Moreover, in the future, we plan to investigate the utility of improving the
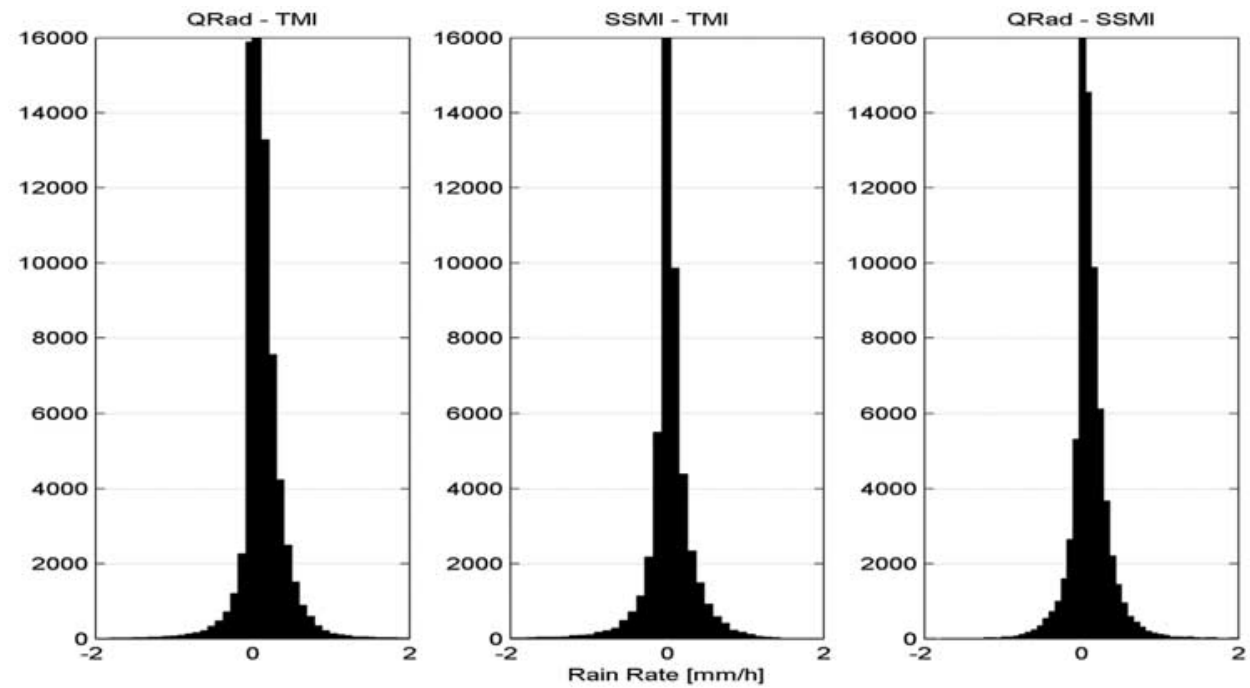

Figure 27. Monthly, global, $0.5^{\circ} \times 0.5^{\circ}$ spatially averaged, rain rate differences for March 2000. From the left are QRad-TMI, SSMI-TMI, and QRad-SSMI. 
Table 8. Monthly Average Rain Rate Differences Between QRad/ TMI, SSMI/TMI, and QRad/SSMI for March 2000

\begin{tabular}{cccc}
\hline Difference & Number of Points & Difference Mean & Difference Std. \\
\hline QRad - SSMI & 75463 & $9.877 \mathrm{e}-2$ & $2.50 \mathrm{e}-1$ \\
QRad - TMI & 75463 & $1.148 \mathrm{e}-1$ & $2.78 \mathrm{e}-1$ \\
SSMI - TMI & 75463 & $1.892 \mathrm{e}-2$ & $2.56 \mathrm{e}-1$ \\
\hline
\end{tabular}

${ }^{\mathrm{a}}$ Units are in $\mathrm{mm} / \mathrm{hr}$.

algorithm by using a microwave radiative transfer model to calculate the daily ocean background instead of using the $T_{b}$ climatology. For this approach, we would use the daily averaged satellite measurements of SST, water vapor and cloud liquid water to remove the inter-annual climatology variability.

[48] 6. Error in estimating the integrated rain rate. Because the TMI integrated rain rate value is not available, the IRR is approximated to be the product of the TMI surface rain rate $(\mathrm{mm} / \mathrm{hr})$ and the rain path length $(\mathrm{km})$. Since both the surface rain rate from the TMI $2 \mathrm{~A} 12$ product and the rain height from the TMI 3A11 product have random errors, this produces increased error in the $\mathrm{T}_{\mathrm{b}}$ - $\mathrm{R}$ empirical relationship. However, the radiometer excess brightness temperature depends upon the integrated rain rate along the propagation path; and because the height of rain varies significantly over latitude, we believe that using this IRR is the best compromise. Further, the TMI training set is distributed over the full latitude range of TRMM, which provides an averaging effect. However, since the QRad algorithm is also applied beyond the latitudinal range of TRMM, caution is advised because of the unknown accuracy in these regions.

\section{Validation of QRad Rain Retrievals}

\subsection{Validation Data Products}

[49] TMI, an improved design of the SSM/I instrument, is dedicated to obtaining quantitative measurements of rainfall. The oceanic instantaneous rain rate, measured by the TMI is widely accepted by the science community to be the best estimate of the true rain rate available from a passive microwave sensor. Thus, to evaluate the $\mathrm{QRad}$ retrieved rain rate capabilities, we use the TRMM 2A12 instantaneous surface rain rate and the TRMM 3B42RT surface rain rates for the comparison data set. The TMI 2A12 instantaneous rain rate product has been validated by the TRMM science team through numerous comparisons with other independent rain measurements [Kummerow et al., 2000]. The TRMM 3B42RT data product [Huffman et al., 2003] available from ftp://aeolus.nascom.nasa.gov/pub/merged combines precipitation estimates in global 3 hour universal time windows ( \pm 90 minute span around synoptic observation hours 00 UTC, 03 UTC, 06 UTC, ..., 21UTC). These estimates are derived from all available high quality (HQ) microwave sources from low earth orbits (three SSM/I's and TMI) combined with visible and infrared rain rate (VAR) estimates derived from geostationary visible/infrared observations. For each $0.25^{\circ}$ grid point the HQ rain rate estimate is used, if available; otherwise the VAR value is used. The 3-hour temporal resolution is driven by the need for the HQ to accumulate a reasonable sample without encompassing a large fraction of the diurnal cycle. The TRMM 3B42RT is based on the Goddard Profiling Algorithm (GPROF) [Kummerow et al., 1996; Olson et al., 1999]. GPROF is a multi-channel physical approach for retrieving rainfall and vertical structure information from passive microwave satellite observations. It applies a Bayesian inversion method to the observed microwave brightness temperatures using an extensive library of cloud model based relations between hydrometeor profiles and microwave brightness temperatures. Each hydrometeor profile is associated with a surface precipitation rate. GPROF includes a procedure that accounts for inhomogeneities of the rainfall within the satellite field of view. The GPROF-SSM/I estimates are computed from the SSM/I satellite data records (SDRs), while the GPROF-TMI estimates are computed by TSDIS as 2A12RT. The GPROF-SSM/I estimates are calibrated and probability matched to 2A12RT. The VAR infrared precipitation estimate are HQ-calibrated locally in time and space. The TRMM 3B42RT rain rates are adjusted to remove bias via histogram matching. Here, we use rain estimates from the TRMM 3B42RT high quality (HQ) microwave sources as the rain "surface truth" for the QRad validation activity.

[50] Also, we use SSMI rain products provided by Remote Sensing Systems http://www.remss.com for quantitative comparisons with the average QRad rain product. SSMI rain products have been validated against independent rain measurements as reported by [Wentz and Spencer, 1998]. Next, we present subjective evaluations for the two QRad precipitation data products.

\subsection{Instantaneous Rain Rates}

[51] A typical instantaneous rain image example is given in Figure 21. The upper panel shows the TMI/QRad near-simultaneous overlapping swaths. Both satellites were in descending revs and observed the rain event with a 20 minute pass time difference. The corresponding rain images are given in the lower panel. For clarity of presentation, both rain images were resampled to $0.125^{\circ}$ resolution. The color bar on the right side indicates the rain rate values $(\mathrm{mm} / \mathrm{hr})$; and both rain images have identical color scales for retrieved rain rates. The shape and intensity of the rain event were well captured by QRad. In fact, the correlation coefficient for the two images is found to be $89.7 \%$.

[52] Additional evaluations of the instantaneous QRad retrieval algorithm consisted of comparisons with the high quality merged TRMM 3B42RT real time multi-satellite precipitation data product. A hundred and eight significant rain events that were observed by QRad and HQ microwave radiometers are used as an additional independent data set for this validation activity. Overall the rain intensity and spatial rain patterns were well captured by QRad and the correlation coefficients between corresponding rain images was typically $>0.70$.

[53] The first quantitative comparison for these hundred and eight rain events is presented as a scatter plot in Figure 22. Statistical results of the differences (QRad minus HQ) are presented in Table 7, where we bin the data in five ranges of HQ rain rate. Although the standard deviations for the individual bins are large due to the poor $\mathrm{QRad} \Delta \mathrm{T}$, the retrievals are well behaved in the mean. 


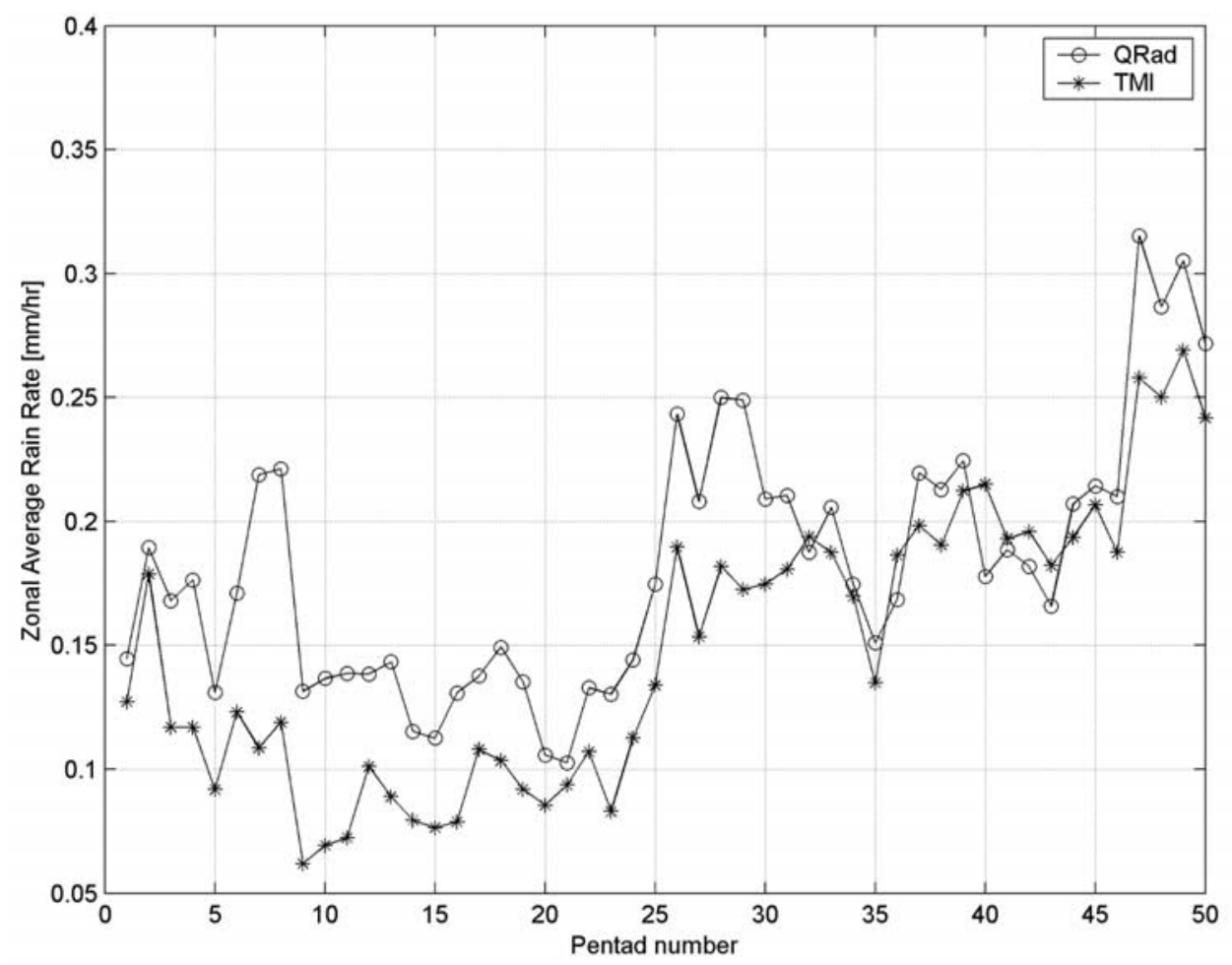

Figure 28. Zonal averages $\left(0^{\circ} \mathrm{N}\right.$ to $\left.20^{\circ} \mathrm{N}\right)$ of five day (pentad) average rain rate for QRad and TMI from January 2000 through September 2000.

[54] The second quantitative comparison for the hundred and eight rain events is presented in terms of the rain rate probability density functions (pdf's) shown in Figure 23. Clearly, the large $\Delta \mathrm{T}$ causes some distortion in QRad pdf for low rain rate values; however, for larger rain rates $>2.5 \mathrm{~mm} / \mathrm{hr}$, the QRad pdf captures the behavior of the HQ rain rate distribution.

[55] Next, we present sample image comparisons of collocated rain events of QRad and HQ retrieved rain rates. Although these collocated rain events are obtained from the 3-hour UTC windows, we utilize a satellite orbit database, along with specialized collocation tools to estimate the overpass time differences between QRad and HQ observations. These collocations span a period of about two weeks during the month of June 2003. First, the QRad rain was put into 3-hour universal time windows $( \pm 90$ minute span around synoptic observation hours 00 UTC, 03 UTC, $06 \mathrm{UTC}, \ldots, 21 \mathrm{UTC})$. Then, the resulting time binned rain images were smoothed by resampling to a $0.25^{\circ} \times$ $0.25^{\circ}$ latitude/longitude grid to match the HQ rain product resolution.

[56] The upper panel in Figure 24 shows a collocated rain event with low rain values that was observed on June 18 , 2003 during the 06 UTC time-window where the coincidence time differences are $<35 \mathrm{~min}$. The QRad rain rates are shown on the right side, while the HQ rain rates are shown on the left side, and the color bars indicate the rain rate $(\mathrm{mm} / \mathrm{hr})$ values. The correlation coefficient between the two images is $85 \%$. The lower panel shows a second collocated rain event with moderate rain values that was observed on June 21, 2003 during the 06 UTC time-window where the coincidence time differences are also $<35 \mathrm{~min}$. For this case, the spatial correlation coefficient is $75 \%$. A third rain image comparison presented in the upper panel of Figure 25 represents an example of high rain rate that was observed on June 24, 2003 during the 15 UTC time-window where the coincidence time differences are $<60 \mathrm{~min}$. The correlation is found to be $80 \%$. The last collocated rain event example is shown in the lower panel of the same figure. This rain event was observed on June 25, 2003 during the 15 UTC time-window where the coincidence time differences are also $<60 \mathrm{~min}$. The correlation coefficient for this event is found to be $85 \%$.

[57] In general, there is very good spatial correlation between QRad and HQ rain patterns. Because of the smaller IFOV and lower $\Delta \mathrm{T}$, the HQ images are "crisper"; nevertheless, the shape and relative intensity of the rain events are well captured by the QRad images. On an absolute basis, the QRad underestimates the higher rain rates because of the non-linear effects of beam filling. Further, the effects of the high $\Delta \mathrm{T}$ result in "noisy pixels" that is apparent in the QRad rain images. Most differences between HQ and QRad are attributed to errors in the QRad retrievals; however some differences may be "real" in that they could be the result of the different pass times of QRad and HQ over the rain events.

\subsection{Averaged Rain Rates}

[58] For the average rain rate product, we perform temporal (pentad) and spatial $\left(0.5^{\circ} \times 0.5^{\circ}\right)$ averaging of all instantaneous rain rate values (positive and negative) which significantly reduces the random component of the rain retrieval. As an example, Figure 26 shows the average rain rate for March 2000, produced from QRad, TMI and SSM/ 

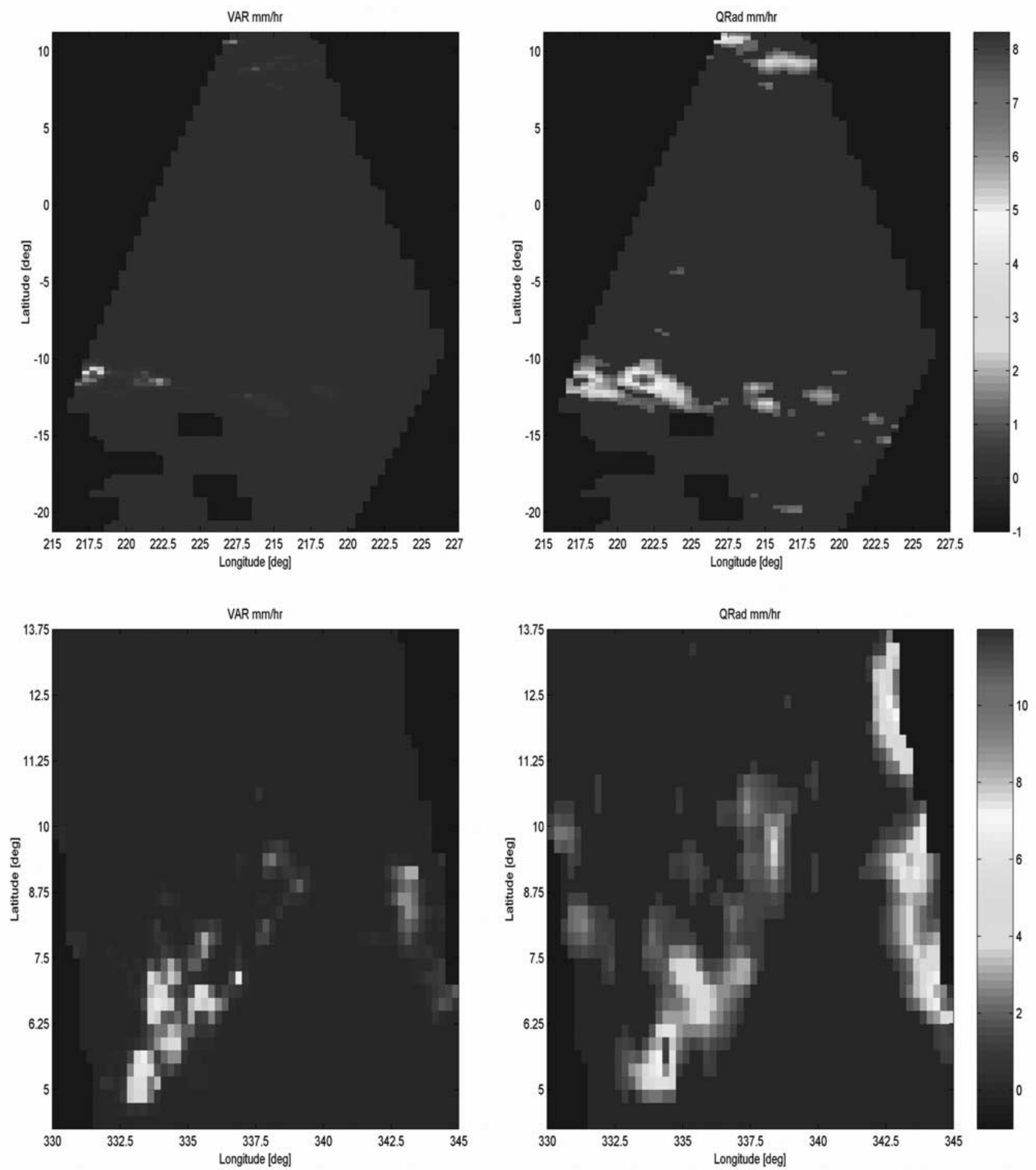

Figure 29. Typical examples of near-simultaneous collocation cases for QRad (right) and TRMM 3B42RT VAR (visible and infrared) product (left). Spatial resolution is $0.25^{\circ}(25 \mathrm{~km})$. See color version of this figure at back of this issue.

I-F13, averaged over the global region $\pm 40^{\circ}$ latitude on a $0.5^{\circ} \times 0.5^{\circ}$ latitude/longitude grid. As the spatial resolution decreases (i.e., spatial averaging area increases), the correlation improves. An example of the differences between the three rain rate retrievals for $0.5^{\circ} \times 0.5^{\circ}$ for March 2000 is presented in Figure 27, and the statistical measures for these cases are given in Table 8. Here there is excellent agreement between TMI and SSMI and quite reasonable comparisons for both with QRad. Most of the difference occurs in the vicinity of the ITCZ area where the convective rain activity predominates.

[59] In general, there is excellent correlation between the spatial patterns of rain; however there are fine scale differences due to the larger spatial resolution of QRad, and its 
poorer radiometric precision $(\Delta \mathrm{T})$. Nevertheless, the shape and the relative intensity of the rain are well captured by QRad.

[60] Finally, Figure 28 shows a time series of QRad and TMI zonal five-day (pentad) rain rates, averaged over the tropical ocean from $0^{\circ} \mathrm{N}$ to $20^{\circ} \mathrm{N}$. Pentad averages were calculated for about nine months during January 2000 through September 2000. Although QRad slightly over estimates the rain rate, there is high correlation between these two time series $(\sim 86 \%)$, and this result is in excellent agreement with a similar study of Imaoka and Spencer [2000] between pentad averages for TMI and SSMI.

\section{Summary}

[61] This paper discussed the details of the QRad statistical rain retrieval algorithm. Comparisons between rain products derived from QRad, and rain retrievals obtained from independent microwave rain measuring instruments are presented. Results demonstrate that QRad rain measurements are in very good agreement with these independent rain estimates in terms of the spatial distribution of the rain patterns and the relative rain intensity. However, due to the poor radiometric resolution $(\Delta \mathrm{T})$ of $\mathrm{QRad}$, some fine scale differences between the rain retrievals are noticed.

[62] When compared to rain measurements obtained from visible and infrared satellite observations, QRad rain estimates perform superbly. As an example, Figure 29 presents two collocated rain events between QRad and the TRMM 3B42RT VAR data product. The QRad rain rates are shown on the right side, while the VAR rain rates are shown on the left side. The color bars are proportional to the rain rate $(\mathrm{mm} / \mathrm{hr})$ values. For these comparisons we apply a threshold of $1 \mathrm{~mm} / \mathrm{hr}$ to QRad rain rates to eliminate any random bogus rain pixels. In both cases, it can be seen that the VAR rain estimates failed to detect a significant portion of the low and moderate rain event structure. These examples are quite typical, and they emphasize the superior performance of the microwave rain retrievals compared to rain estimates from visible and infrared sources.

[63] The major scientific utility of QRad rain measurements is that they provide additional independent temporal and spatial sampling of the oceanic rain, which complements the coverage provided by TMI and the SSMIs' instruments. Thus the QRad rain time series from 1999 to present is a valuable addition to the oceanic precipitation climatology data set that can be potentially used to improve the diurnal estimation of the global rainfall, which is a goal for the future Global Precipitation Mission program. Moreover, the early availability of QRad data will afford users early access to learn to use less-precise rain measurements that will occur in the future with the use of less-capable constellation satellites. Finally, these QRad rain estimates will be available in the planned data reprocessing (FY 2006) to users of QuikSCAT winds to improve the rain flagging of rain-contaminated oceanic wind vector retrievals.

[64] Acknowledgments. This work was sponsored by NASA's Earth Science Enterprise, jointly by the TRMM Project at NASA GSFC and by the QuikSCAT Project at the Jet Propulsion Laboratory. The authors wish to thank Deborah Smith and Carl Mears (Remote Sensing Systems) for their support in this research. SSM/I data are produced by Remote Sensing Systems and sponsored by the NASA Earth Science REASoN DISCOVER Project. Data are available at http://www.remss.com.

\section{References}

Ahmad, K. A., W. L. Jones, and T. Kasparis (2003), Precipitation measurements using the QuikSCAT Radiometer, paper presented at International Geoscience and Remote Sensing Symposium, Inst. of Electr. and Electron. Eng., Toulouse, France, 21-25 July.

Bell, T. L., and N. Reid (1993), Detecting the diurnal cycle of rainfall using satellite observations, J. Appl. Meteorol., 32(2), 311-322.

Chang, A. T. C., L. S. Chiu, and G. Yang (1995), Diurnal cycle of oceanic precipitation from SSM/I data, Mon. Weather Rev., 123(11), 3371-3380. Huffman, G. J., R. F. Adler, E. F. Stocker, D. T. Bolvin, and E. J. Nelkin (2003), Analysis of TRMM 3-hourly multi-satellite precipitation estimates computed in both real and post- real time, paper presented at 12th Conference on Satellite Meteorology and Oceanology, Long Beach, Calif., 9-13 Feb.

Imaoka, K., and R. W. Spencer (2000), Diurnal variation of precipitation over the tropical oceans observed by TRMM/TMI combined with SSM/I, J. Clim., 13(23), 4149-4158.

Jones, W. L., R. Mehershahi, and J. Zec (2000), SeaWinds on QuikSCAT radiometric measurements and calibration, paper presented at International Geoscience and Remote Sensing Symposium, Inst. of Electr. and Electron. Eng., Honolulu, Hawaii, 24-28 July.

Kummerow, C., W. S. Olson, and L. Giglio (1996), A simplified scheme for obtaining precipitation and vertical hydrometeor profiles from passive microwave sensors, IEEE Trans. Geosci. Remote Sens., 34, 1213-1232.

Kummerow, C., et al. (2000), The status of the Tropical Rainfall Measuring Mission (TRMM) after two years in orbit, J. Appl. Meteorol., 39(12), 1965-1982.

Mears, C., et al. (2000), QuikSCAT brightness temperatures and their impact on rain flagging, Remote Sens. Syst. Tech. Rep. 031300, Remote Sens. Syst., Santa Rosa, Calif.

Mehershahi, R. J. (2000), Ocean Tb measurements using the QuikSCAT radiometer, M.S. thesis, Univ. of Cent. Fla., Orlando.

Olson, W. S., C. D. Kummerow, Y. Hong, and W. K. Tao (1999), Atmospheric latent heating distributions in the tropics derived from satellite passive microwave radiometer measurements, J. Appl. Meteorol., 38, $633-664$.

Petty, G. W., and K. B. Katsaros (1992), Nimbus-7 SMMR precipitation observations calibrated against surface radar during TAMEX, J. Appl. Meteorol., 31(6), 489-505.

QuikSCAT (2001), Science data product user's manual, Jet Propul. Lab., Pasadena, Calif

Smith, E. (2001), Current development of Global Precipitation Mission (GPM), paper presented at 7th International Conference on Precipitation, Rockport, Maine, 30 June to 3 July.

Smith, E., et al. (2001), Optimizing orbit-instrument configuration for Global Precipitation Mission (GPM) satellite fleet, paper presented at International Geoscience and Remote Sensing Symposium, Inst. of Electr. and Electron. Eng., Sydney, Australia, 9-13 July.

Spencer, M. W., C. Wu, and D. G. Long (1997), Tradeoffs in the design of a spaceborne scanning pencil beam scatterometer: Application to SeaWinds, IEEE Trans. Geosci. Remote Sens., 35, 115-126.

Wang, Y. (2001), A statistical algorithm for inferring rain rate from the QuikSCAT Radiometer, M.S. thesis, Univ. of Cent. Fla., Orlando.

Wentz, F. J., and R. W. Spencer (1998), SSM/I rain retrievals within a unified all-weather ocean algorithm, J. Atmos. Sci., 55, 1613-1627.

Wilheit, T. T., A. T. C. Chang, and L. S. Chiu (1991), Retrieval of monthly rainfall indices from microwave radiometric measurements using probability distribution functions, J. Atmos. Oceanic Technol., 8(1), 118-136.

Wisler, M. M., and J. P. Hollinger (1977), Estimation of marine environmental parameters using microwave radiometric remote sensing systems, NRL Memo. Rep. 3661, Nav. Res. Lab., Washington, D. C., Nov.

I. S. Adams, K. A. Ahmad, W. L. Jones, T. Kasparis, J. D. Park, and S. W. Vergara, Central Florida Remote Sensing Laboratory, Department of Electrical and Computer Engineering, University of Central Florida, P. O. Box 162450, Orlando, FL 32816, USA. (kh393312@pegasus.cc.ucf.edu) 


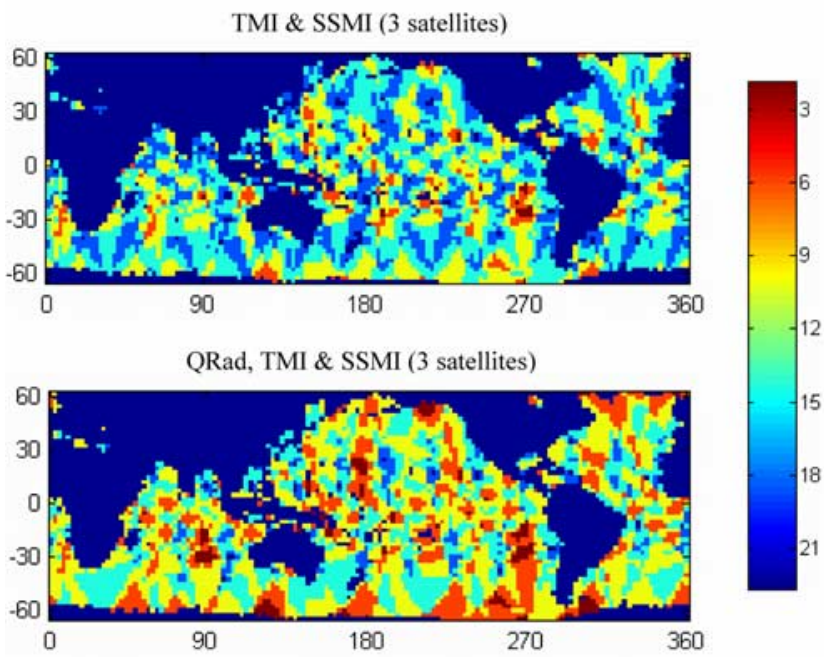

Figure 2. Ocean sampling, daily average revisit time. (top) TMI and 3-SSMI's and (bottom) sampling with QRad added.

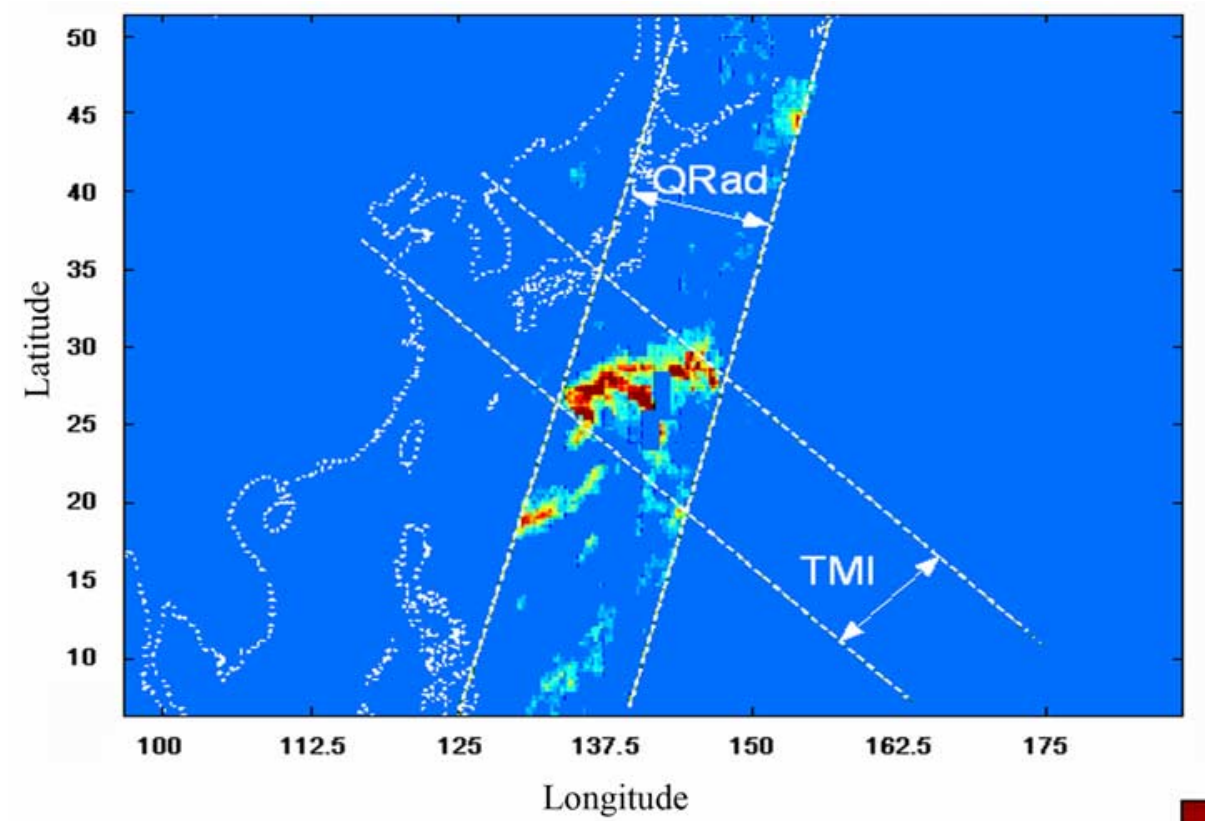

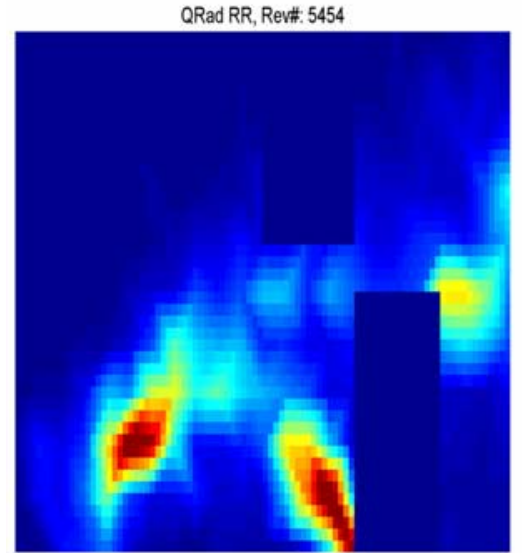

QRad

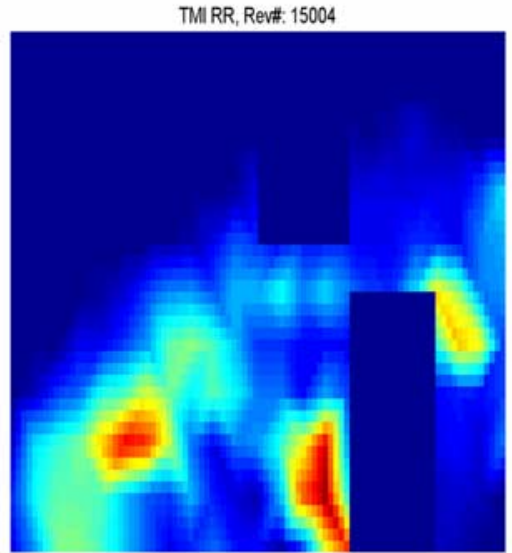

TMI

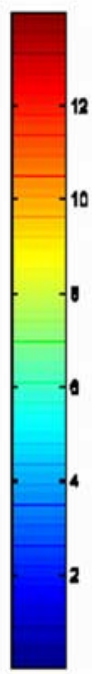

Figure 21. Example of instantaneous rain rate images produced by QRad and TMI. Spatial sampling is $0.125^{\circ}(12.5 \mathrm{~km})$, and coincidence time difference is $\sim 20 \mathrm{~min}$. 

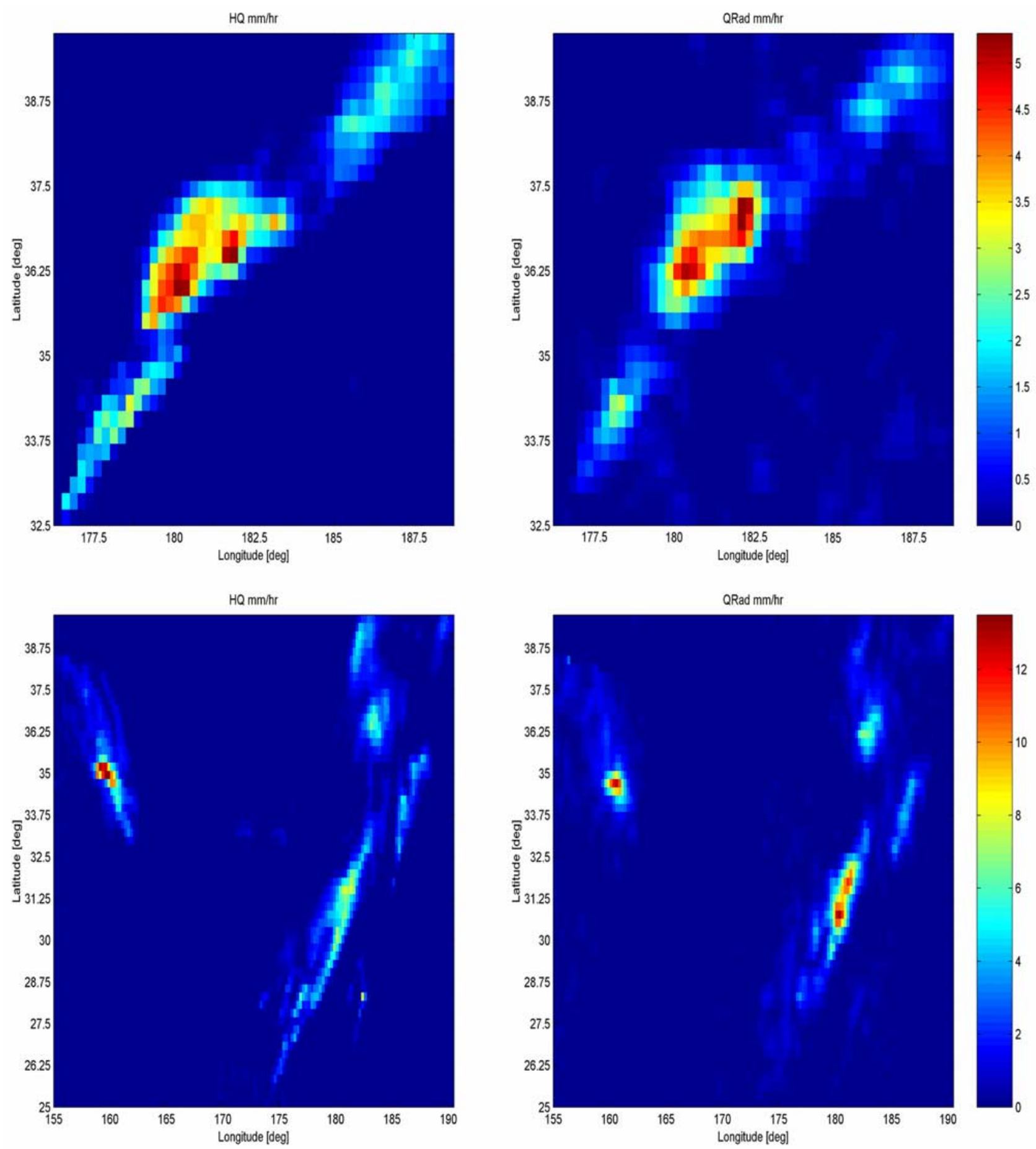

Figure 24. Examples of rain events measured by QRad (right) and TRMM 3B42RT HQ (TMI and $\mathrm{SSM} / \mathrm{I})$ product (left). Spatial resolution is $0.25^{\circ}(25 \mathrm{~km})$ and coincidence time difference $<35 \mathrm{~min}$. 

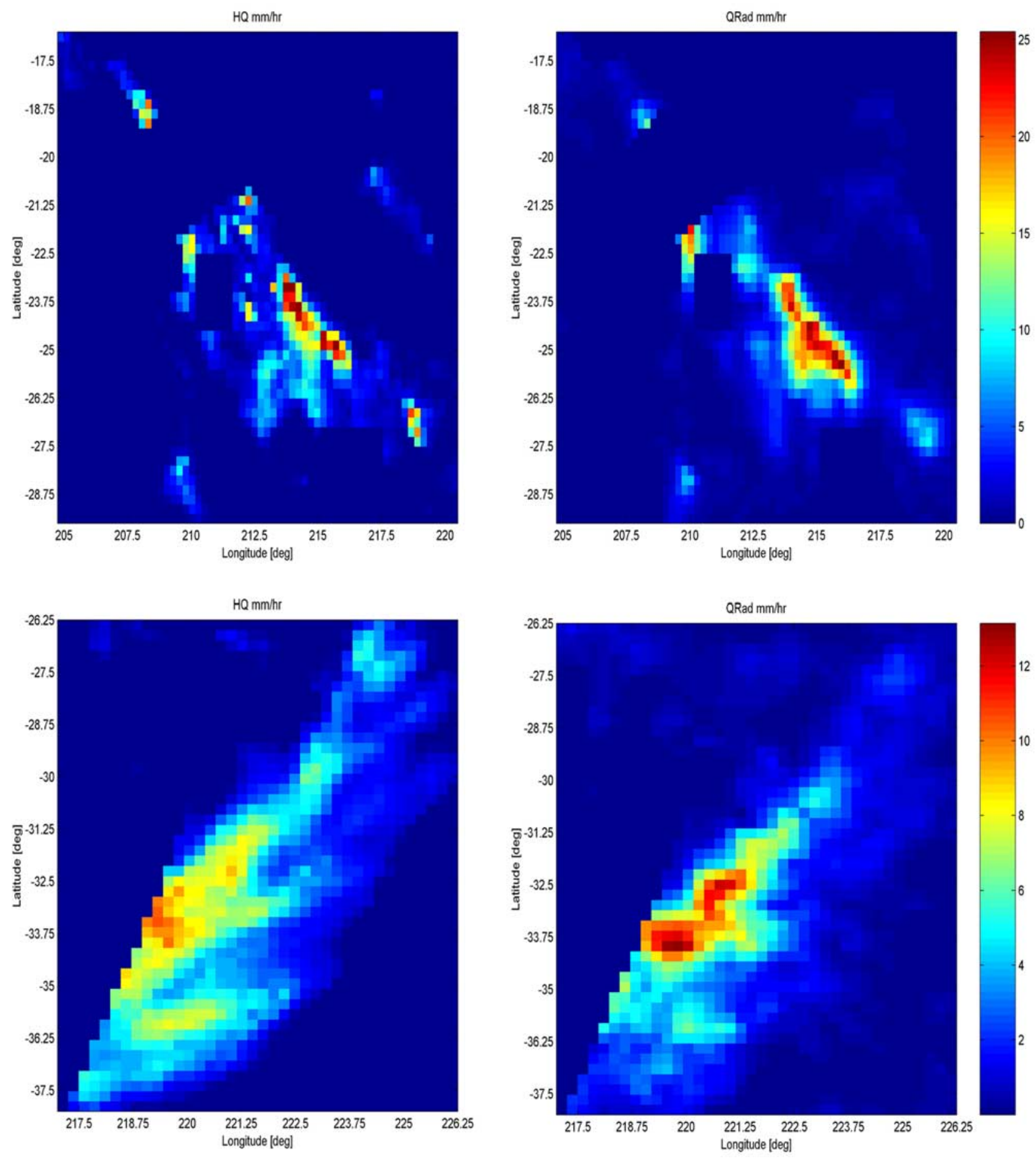

Figure 25. Other examples of rain events measured by QRad (right) and TRMM 3B42RT HQ (TMI and $\mathrm{SSM} / \mathrm{I})$ product (left). Spatial resolution is $0.25^{\circ}(25 \mathrm{~km})$ and coincidence time difference $<60 \mathrm{~min}$. 

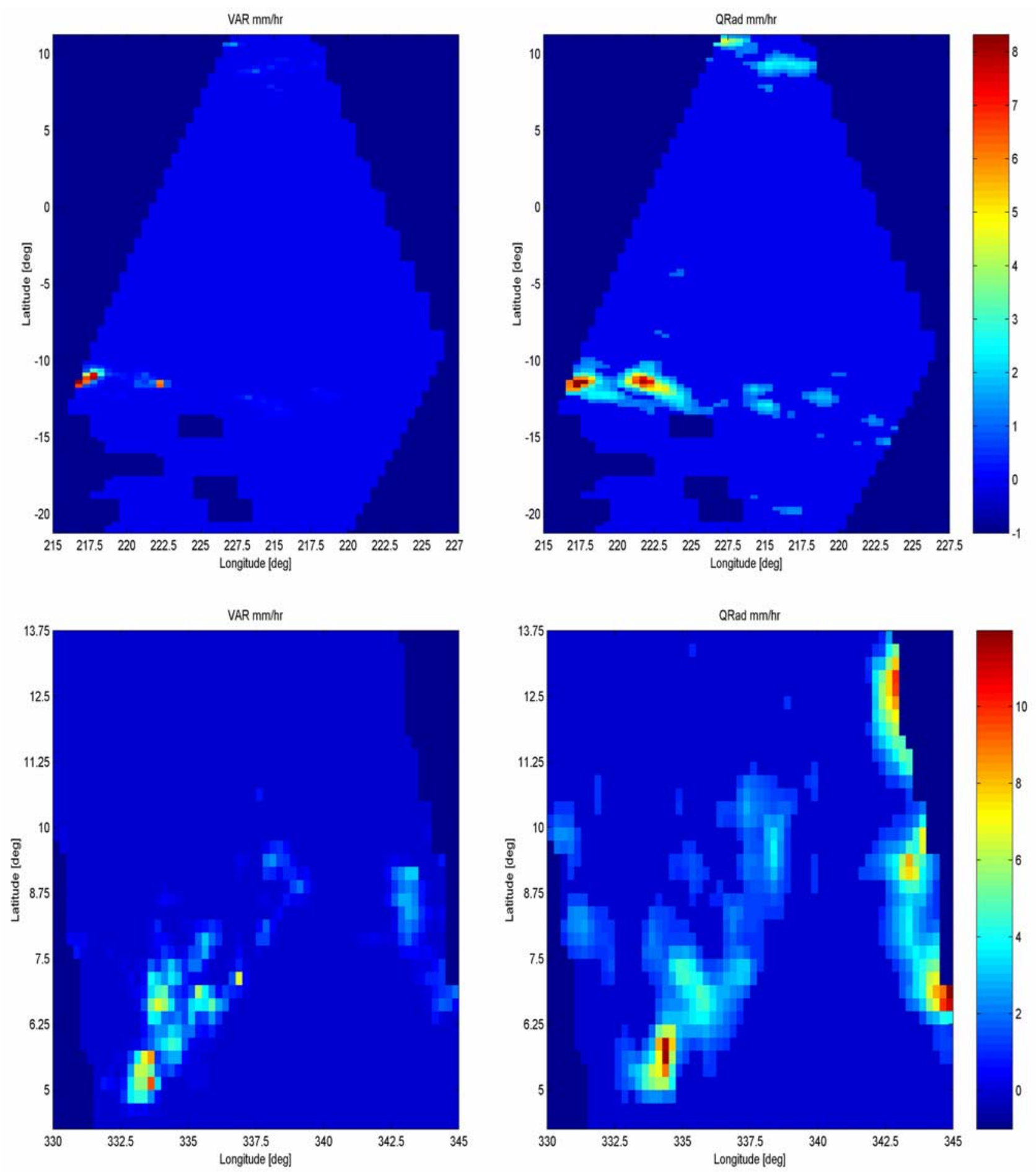

Figure 29. Typical examples of near-simultaneous collocation cases for QRad (right) and TRMM 3B42RT VAR (visible and infrared) product (left). Spatial resolution is $0.25^{\circ}(25 \mathrm{~km})$. 